Artículos 



\title{
PREFERENCIA TEMPORAL, MÚLTIPLES TIPOS DE INTERÉS Y LA TEORÍA AUSTRIACA DEL CICLO ECONÓMICO
}

\author{
ALEJANDRO J. ZAMORA ${ }^{1}$
}

Fecha de recepción: 18 de septiembre de 2015.

Fecha de aceptación: 12 de marzo de 2016.

Resumen: Las exposiciones tradicionales de la Teoría Austriaca del Ciclo Económico (TACE) se apoyan en la referencia a un único tipo de interés de equilibrio. Este artículo, por un lado, explora la cuestión de la validez teórica de dicha referencia $y$, por otro, estudia si ésta es realmente esencial a la TACE. Para analizar dichas cuestiones, partimos de una defensa de la recientemente cuestionada Teoría de la Preferencia Temporal Pura (TPTP), teoría que pretende explicar la causa del fenómeno del interés y que sirve de fundamento a la TACE. A la luz de nuestro estudio, en primer lugar, rechazamos ciertas formulaciones de la TPTP que conducen a interpretaciones erróneas de la misma, proponiendo una enunciación de la teoría que evita confusiones comunes; segundo, negamos la validez de la referencia a un tipo de interés único y su carácter esencial a la TACE; por último, notamos la necesidad de actualizar la exposición de la TACE conforme a las conclusiones antedichas.

Palabras clave: Teoría Austriaca del Ciclo Económico, Ciclos Económicos, Teoría de la Preferencia Temporal Pura, Tipos de Interés, Estructura de Tipos de Interés.

Clasificación JEL: E32, E40, E43, E50, B53.

Title: Time preference, multiple rates of interest and Austrian business cycle theory

\footnotetext{
${ }^{1}$ Máster en Economía por la Universidad Rey Juan Carlos. Licenciado en Derecho por ICADE. Licenciado en Administración y Dirección de Empresas por ICADE. Email: alejandrojzamora@gmail.com
} 
Abstract: Traditional expositions of the Austrian Business Cycle Theory (ABCT) rest on the reference to only one - equilibrium - interest rate. This paper, on the one hand, explores the theoretical validity of such a reference and, on the other hand, analyses whether it is essential to the ABCT. To address both issues, we depart from a defense of the recently-disputed Pure Time Preference Theory (PTPT), which purports to explain the cause of the phenomenon of interest and serves as a basis for the ABCT. In the light of our study, in the first place, we reject some of the formulations of the PTPT that lead to erroneous interpretations of this theory and we put forward an enunciation that avoids common confusions; secondly, we deny the validity of the reference to only one interest rate as well as its essentiality to the $A B C T$; finally, we point out the necessity of updating the exposition of the $A B C T$ in consonance with the previous conclusions.

Key words: Austrian Business Cycle Theory, Business Cycles, Pure Time Preference Theory, Interest Rates, Term Structure of Interest Rates.

JEL Classification: E32, E40, E43, E50, B53.

\section{I \\ INTRODUCCIÓN}

No es arriesgado describir la Teoría de la Preferencia Temporal Pura (TPTP) como el puente analítico que une el fenómeno (aparentemente monetario) del tipo de interés con el de determinados cambios en la estructura productiva (de naturaleza indiscutiblemente real). Es por ello que esta teoría constituye un importante pilar sobre el que se fundamenta la Teoría Austriaca del Ciclo Económico (TACE). En consecuencia, aquellos que se propongan arrojar una crítica exitosa contra la TACE, bien pueden seguir la estrategia de impugnar primero las enseñanzas de la TPTP $y$, concretamente, la noción del tipo de interés descrita por ella. Hasta ahora, empero, todos los intentos de esta suerte han sido estériles. Entre estas críticas, las más reseñables son dos: la primera, la que propugna la incompatibilidad de la TPTP con la teoría subjetiva del valor que los propios economistas austriacos defienden; la segunda, la que discute la referencia a un tipo de interés único, que juzga inútil la TACE tras creer demostrar la invalidez de esta referencia. El objetivo más importante de este escrito es afrontar la 
segunda de las críticas, es decir, la que pretende desmontar la TACE mediante la objeción a la referencia a un único tipo de interés; expondremos cómo dicha referencia, en efecto, es teóricamente inválida, pero nos opondremos a considerar que el admitir tal crítica exija descartar de plano la TACE, puesto que, como argumentaremos, dicha alusión a un único tipo de interés no es esencial a esta teoría. Para abordar adecuadamente estas cuestiones, sin embargo, no podemos omitir una respuesta a la primera de las críticas, que cuestiona la validez de la TPTP. Argüiremos que, a nuestro parecer, dichas críticas no se dirigen, en realidad, contra la TPTP, sino contra ciertas enunciaciones de la misma que, si no son defectuosas, sí son harto confusas. Por ello, concluiremos que el lenguaje de algunas de las exposiciones tradicionales de la TPTP debe ser corregido. Será, por tanto, ésta la primera cuestión que abordaremos en el presente artículo. Solo un entendimiento previo y adecuado de ella nos permitirá abordar la materia, ciertamente indócil, de la referencia a un tipo de interés único en la exposición tradicional de la TACE.

Hemos estructurado estos objetivos de la siguiente forma: en el epígrafe II, exploramos las causas del fenómeno del interés e incidimos en la necesidad de rechazar ciertas enunciaciones tradicionales de la TPTP que han conducido a interpretaciones erróneas de esta teoría, para después proponer una formulación de la teoría de la mano de Heberner (2011) que, creemos, evita gran parte de las confusiones comunes. El objetivo referido al estudio de la alusión a un único tipo de interés único en la TACE nos ocupará los dos siguientes epígrafes: en el epígrafe III examinamos el célebre debate entre Hayek (1967 [1931], 1932) y Sraffa (1932a, 1932b) sobre la referencia a un tipo de interés único y, en relación con esto, la propuesta de Murphy (2010), quien lamenta que los austriacos no hayan conseguido - ni hayan tenido voluntad- de incorporar las críticas de Sraffa a la TACE; en el epígrafe IV abordamos las cuestiones, por un lado, de la realidad o validez de la referencia al tipo de interés único en la TACE, y por otro, de si dicha referencia es en realidad esencial a esta teoría; para acabar este epígrafe, hacemos una breve pero necesaria alusión al patrón de la curva de rendimientos a lo largo del ciclo. Finalmente, en el epígrafe $\mathrm{V}$ concluimos. 


\section{II \\ UNA REAFIRMACIÓN DE LA TEORÍA DE LA PREFERENCIA TEMPORAL PURA}

Es conocido el problemático hecho económico que las teorías del interés pretenden explicar, a saber, el agio existente entre el precio del producto y el precio de los factores de producción que aparece incluso en equilibrio, es decir, cuando el empresario no obtiene beneficios empresariales puros sin incurrir en pérdidas. La razón inmediata de este hecho en una economía monetaria parece evidente $y$, de hecho, es indiscutida: una unidad monetaria futura ha de valer menos que esa misma unidad monetaria en el presente si eliminamos el efecto del cambio en el poder adquisitivo de la misma. La verdadera dificultad con la que tropiezan los teóricos de la economía aparece al inquirir por qué una unidad monetaria en el presente es más valorada que la misma en el futuro. Sólo investigando esta cuestión se pueden definir las verdaderas causas del fenómeno del interés. Los defensores de la TPTP buscan explicar el antedicho fenómeno económico de una forma fundamental, apoyándose en la idea de que el valor que el hombre asigna al tiempo es una condición esencial de la acción humana. A pesar de lo diáfano de esta idea, la TPTP ha recibido un importante número de críticas, no sólo por autores que (presumiblemente) no suelen visitar la literatura austriaca, sino también por parte de economistas adheridos a esta escuela ${ }^{2}$. A nuestro parecer esto refleja, por un lado, que algunas de las formulaciones de la TPTP no son tan claras como deberían, y por otro, que las críticas a la teoría no se han respondido con la solidez necesaria. Dada la posición central que ocupa esta teoría en el corpus austriaco (y, en particular, por ser un pilar básico de la TACE), creemos que no es baladí enfocar nuestros esfuerzos en enunciar la TPTP de forma más depurada y limpia de las confusiones que acarrean las formulaciones tradicionales. Para ello no es necesario realizar ninguna innovación sobre el contenido de la teoría, sino sólo enmendar su formulación tradicional, ya que el núcleo de nuestra exposición deriva del tratamiento praxeológico que Mises dio a la teoría. (2003).

${ }^{2}$ Notablemente, Pellengahr (1996), Reisman (1996), Hülsmann (2002) y Murphy 


\section{El valor del tiempo}

La TPTP parte del hecho indiscutido de que el hombre valora el tiempo. Como dice Mises, el tiempo para el hombre es escaso e irreversible y, por tanto, debe economizarlo como el resto de bienes escasos $^{3}$. Pero la economización del tiempo se manifiesta de forma diferente que la economización del resto de bienes ${ }^{4}$. Es evidente que, al ser incapaz de realizar todos los fines que desea en el momento presente ${ }^{5}$, el hombre se ve obligado a distribuir dichos fines a lo largo del tiempo. No obstante, esta proyección de fines a lo largo del tiempo viene dada por la incapacidad del hombre de satisfacer todos ellos en el momento presente, pero ello no refleja el valor que el hombre asigna al tiempo. Lo único que refleja es que, dada la imposibilidad de satisfacer todos ellos en el momento presente, prefiere que determinados fines sean resueltos antes que otros. Por lo tanto, hay fines presentes que valora más que otros fines futuros; pero también es cierto decir que hay fines cuya satisfacción prefiere que se realice en el futuro, ya que hay otros que desea satisfacer más inmediatamente.

Como decimos, toda esta distribución de fines a lo largo del tiempo no es, en realidad, una manifestación del valor que da el hombre al tiempo o, en otras palabras, no es un reflejo de cómo el hombre economiza el tiempo. Es, sencillamente, la única forma que el hombre tiene de actuar dada la condición limitante de la acción de no poder satisfacer en el presente todos los fines que en ese momento desea. El valor que el hombre da al tiempo se manifiesta, en cambio, en la voluntad de satisfacer todos los fines cuanto antes. $\mathrm{O}$ dicho de otro modo quizá más claro: de todos aquellos fines que el hombre, en un determinado momento, quiere ver realizados,

\footnotetext{
${ }^{3}$ «Man is subject to the passing of time... His time is scarce. He must economize it as he does other scarce factors». Mises (1998, p. 101).

4 «The economization of time has a peculiar character because of the uniqueness and irreversibility of the temporal order». Mises (1998, p. 101).

5 "Two actions of an individual are never synchronous; their temporal relation is that of sooner and later (...) A man's individual actions succeed one another. They can never be effected at the same instant; they can only follow one another in more or less rapid succession». Mises (1998, p. 102).
} 
quiere verlos realizados cuanto antes ${ }^{6}$. Como hemos dicho, habrá fines (o expectativas de fines) que preferirá realizar en el futuro y no en el presente, porque las condiciones presentes de la acción no son tales que hagan que el fin sea más inmediatamente deseado que otros fines. Pero, insistimos, esto no corresponde a la valoración que el sujeto hace del tiempo, sino más bien a la proyección de los posibles fines a realizar en el futuro. Separar estas dos ideas parece obligado para entender adecuadamente la TPTP que se basa sólo en una de ellas: el valor que el hombre asigna al tiempo, y no la distribución de fines a lo largo del tiempo. Lo único que supone la primera de estas ideas es, entonces, que una vez surgida la necesidad inmediata del fin, el valor que asigna el hombre al tiempo hace que éste trate de satisfacer dicho fin mejor antes que después. Esto es, si tomamos el fin que más inmediatamente quiere satisfacer en su particular distribución de fines a lo largo del tiempo, el hombre querrá verlo satisfecho cuanto antes. Es ésta simple idea la que sirve de fundamento a la TPTP.

\section{Los componentes de las valoraciones intertemporales}

\section{a) El difícil matrimonio entre la TPTP y la teoría subjetiva del valor}

Una de las formas más extendidas y a la vez más desafortunadas de expresar la idea de que el hombre valora el tiempo (y que, por ello, prefiere satisfacer sus fines inmediatos con la mayor inmediatez posible) es decir que el hombre, en igualdad de condiciones, siempre prefiere un bien presente a ese mismo bien en el futuro; $\mathrm{o}$,

\footnotetext{
${ }^{6}$ Nótese que no hay nada de tautológico en esta idea. No se dice que los fines que el hombre desea de manera inmediata, los desea de manera inmediata; se dice que los fines que el hombre desea de manera inmediata, los desea con la mayor inmediatez posible - es decir, cuanto antes-.

${ }^{7}$ Como dice Mises (1998, p. 476), «Acting man distinguishes the time before satisfaction of a want is attained and the time for which the satisfaction continues... Between the setting in of action and the attainment of the end sought there always elapses a fraction of time, viz., the maturing time in which the seed sown by the action grows to maturity». Es este ínterin hasta que se realiza el fin el que el hombre desea reducir para, así, economizar el tiempo.
} 
similarmente, que en igualdad de condiciones, el hombre preferirá en todo caso una satisfacción en el presente a la misma satisfacción en el futuro.

No es sorprendente, a nuestro juicio, que dicha formulación induzca a confundir las dos ideas expuestas en el apartado anterior - el valor del tiempo y la necesidad de distribuir los fines en el tiempo-, cuando en realidad la TPTP se refiere sólo a una de ellas - el valor del tiempo- - Si ambas se confunden, parece natural creer que hay determinadas satisfacciones que son más intensamente deseadas en el futuro que en el presente (lo cual abriría la posibilidad de tipos de interés negativos). Sólo aislando el componente de la valoración intertemporal que se refiere al valor del tiempo, se puede llegar a la conclusión de que el sujeto prefiere que sus fines sean satisfechos antes que después. La raíz del problema, creemos, está en el desafortunado empleo de la locución «en igualdad de condiciones» para enunciar la teoría. La pregunta inmediata que uno debe hacerse es qué condiciones deben estar en igualdad para realizar la comparación entre bien presente y bien futuro, y la respuesta a dicha pregunta no es del todo evidente. La complicación radica en que esta formulación de la TPTP, utilizando la cláusula caeteris paribus, parece colisionar y ser difícilmente reconciliable con la teoría subjetiva del valor que los austriacos defienden. En la exposición de otras leyes económicas, la utilización de la cláusula caeteris paribus no supone ninguna inconsistencia analíti$\mathrm{ca}^{8}$. En efecto, si decimos que, caeteris paribus, un aumento de la cantidad demanda de una mercancía hace incrementar su precio, dicha ley congela los efectos del resto de variables presentes permitiendo abstraer la relación causa-efecto a analizar. Sin embargo, resulta más complicado decir lo mismo para una ley que trata de explicar la preferencia subjetiva entre dos bienes en distintos momentos de tiempo. Así, si queremos congelar el resto de variables para abstraer el valor que el hombre asigna al tiempo en la comparación entre dos bienes, estaríamos anulando de inmediato

${ }^{8}$ A pesar de que la correcta enunciación de las mismas no habría de limitarse a describir la relación causa-efecto en cuestión cuando las circunstancias permanecen inalteradas, sino a pesar de que las circunstancias se alteren, lo cual se consigue elegantemente indicando su naturaleza contrafáctica (Hülsmann 2003, pp. 74-75). 
las enseñanzas de la teoría subjetiva del valor. En otras palabras, en el caso de la ley de la demanda es posible suprimir el efecto de las demás leyes para abstraer el efecto del que versa la primera; sin embargo, resulta confuso exponer la ley de la preferencia temporal anulando la teoría subjetiva del valor ya que ésta es la base de todas las valoraciones que realiza el sujeto, y la TPTP entraña una valoración. Si el sujeto tiene que hacer la comparación entre una satisfacción en el presente y la «misma» satisfacción en el futuro, se topa con que en el futuro prevalecerán condiciones diferentes a las del presente, irremediablemente haciendo que las satisfacciones sean, por tanto, diferentes. Entonces, decir que el sujeto puede comparar la preferencia de una satisfacción en el presente con la misma satisfacción en el futuro «en igualdad de circunstancias», es una contradicción en los términos, no sólo desde el punto de vista objetivo (las condiciones espacio-temporales son distintas) sino, más importante, desde el punto de vista subjetivo (las condiciones son, o al menos pueden ser, percibidas distintas). Como dice Hülsmann (2002):

[This] approach ran into even greater conflict with the theory of subjective value insofar as it claimed that time preference concerns the value differential between homogenous present and future goods. The very fact, however, that two goods exist at different points of time makes them heterogeneous goods (...) The point is devastating for the old time preference theory, for one cannot even make claims on behalf of present and future goods «of the same economic quality» without contradicting oneself.

(Hulsmann 2002, P. $82^{910}$ )

\footnotetext{
${ }^{9}$ Hülsmann, en su artículo, defiende que Mises supera esta formulación con una exposición contrafáctica de la TPTP (Hülsmann 2002, p. 83). Sin embargo, como veremos enseguida, Mises en ocasiones sigue utilizando la misma formulación que sus predecesores, sin terminar de aclarar sus contradicciones.

${ }^{10}$ Como nota Hulsmann (2002), Pellengahr (1996) también ha señalado esta contradicción de forma muy elocuente: «[I]t is left entirely unclear how the evaluating agent is to separate his decision with respect to the equality of the satisfactions from the decision as to which satisfaction he prefers. The task is simply too difficult for anyone to perform: either two satisfactions, one present, one future, are considered equal or not. They cannot, qua satisfactions, be considered equal in one respect and unequal in another. A concept of time preference defined with respect to equal present
} 
En resumen, los defensores de la TPTP, en su empeño de enunciar la teoría utilizando la locución caeteris paribus, llegan a un callejón sin aparente salida. El crítico no puede entender cómo los defensores de la TPTP pretenden asumir que una satisfacción en un momento puede ser la misma satisfacción si se realiza en otro momento (lo que es contrario a la teoría subjetiva del valor) y, al propio tiempo, colegir que en el caso de que esas dos satisfacciones sean idénticas, existe para el sujeto un diferencial de valor positivo. Como veremos, esta contradicción es solo aparente.

Los partidarios de la TPTP han tenido, en diversas ocasiones, la oportunidad de aclarar tales críticas y han respondido obstinadamente de la misma forma insatisfactoria en todas ellas. Tal vez una de las formas más burdas, pero también más famosas, de argumentar que sí es posible que los sujetos valoren más intensamente un bien presente que el mismo bien en el futuro es el intento de contraejemplo en el que el sujeto, en invierno, compara el hielo estival (futuro) con el hielo invernal (presente). Este ejemplo es utilizado por los teóricos de la preferencia temporal pura para aclarar el significado de la teoría (Mises 1998, pp. 486-7; Rothbard 2004, pp. 15-6; Block 1978, pp. 121-2), y en todos los intentos de aclaración estos autores han desperdiciado la oportunidad de desechar la problemática enunciación de la TPTP a la que nos referimos ${ }^{11}$. Concretamente, la solución que ofrecen los defensores de la TPTP a los casos en que el bien hielo futuro puede ser más valorado que el bien hielo presente es que, sencillamente, en este caso, dichos bienes (o satisfacciones) no son los mismos, y por tanto no es posible deducir un diferencial valorativo positivo entre ambos. En lo que

and future satisfactions, however alluring it may seem to subjectivists at first glance because of its apparent subjectivism, is thus simply a contradiction in terms». (Pellengahr 1996, pp. 26-27).

${ }^{11}$ Quizás es Rothbard quien más clara y concisamente afronta esta crítica: «[A] common type of objection to the assertion of universal time preference is that, in the wintertime, a man will prefer the delivery of ice the next summer (future) to delivery of ice in the present. This, however, confuses the concept "good" with the material properties of a thing, whereas it actually refers to subjective satisfactions. Since ice-inthe-summer provides different (and greater) satisfactions than ice-in-the-winter, they are not the same, but different goods. In this case, it is different satisfactions that are being compared, despite the fact that the physical property of the thing may be the same». (Rothbard 2004, pp. 15-6) 
no parecen reparar Mises, Rothbard y Block al negar la posibilidad de observar este diferencial positivo al hacer comparaciones intertemporales entre dos satisfacciones distintas es que, diciendo esto, limitan excesivamente la aplicación de la TPTP, de tal suerte que su formulación tradicional («el hombre valora, en igualdad de condiciones, los bienes presentes a los bienes futuros») se vacía de contenido. En puridad, como hemos señalado, cualquier par de bienes situados en dos momentos diferentes de tiempo - aunque sean físicamente idénticos-son bienes diferentes desde un punto de vista económico. Por tanto, no cabría deducir un mayor valor de bienes presentes respecto a bienes futuros salvo en el caso de que, efectivamente, el sujeto valorase más los bienes presentes que los bienes futuros. Pero en tal caso, la universalidad de la ley se tambalea y se abriría la posibilidad de que existiesen en ocasiones tipos de interés negativos. En suma, si llevamos la respuesta de Mises (si los bienes son diferentes, no se puede deducir en todo caso un diferencial de valor positivo entre el bien presente y el bien futuro) a sus últimas implicaciones lógicas, la formulación de que el hombre preferirá, en igualdad de condiciones, la satisfacción presente a la misma satisfacción en el futuro, pierde todo su contenido (ya que según la teoría subjetiva del valor, una misma satisfacción en el presente y en el futuro es, o al menos puede ser, a los ojos del actor, diferente).

\section{b) La solución fetteriana}

Comprobamos que el origen de estas críticas a la TPTP se encuentra en una confusa enunciación de la misma que sacrifica la exactitud científica por la sencillez lingüística. Lo que Mises y sus discípulos en realidad quieren decir es que, dada una satisfacción inmediata, es preferible para el sujeto que el periodo desde que se inicia la acción hasta que se obtiene dicha satisfacción sea lo más corto posible. Y esto ocurre porque los hombres en todo caso valoran positivamente el tiempo, como hemos expuesto en el primer apartado de este epígrafe. Pero sin comprender el origen de su razonamiento (que no siempre especifican) es difícil entender tan confusa formulación. La conclusión a la que éstos llegan, es que, si 
este valor positivo que el hombre asigna al tiempo aparece en todo caso, o dicho de otro modo, es una categoría esencial de la acción humana, entonces tiene que aparecer también en cualquier comparación intertemporal. Por lo tanto, cuando dicen «en igualdad de condiciones», lo que en realidad quieren decir es «suprimiendo cualquier otra diferencia valorativa que pueda surgir en la comparación entre una satisfacción en el presente y lo que el sujeto reputa que es la misma satisfacción en el futuro». Si se prefiere satisfacer un fin en el futuro y no el presente, esto no sucede porque el actor, en ese caso, valore el tiempo de manera negativa; sucede porque, como hemos argumentado al principio de este epígrafe, en la distribución a lo largo del tiempo que el hombre hace de los fines que quiere satisfacer existen otros fines cuya satisfacción desea más inmediatamente. Dadas las condiciones presentes y las expectativas de las condiciones futuras, por tanto, el hombre distribuye sus fines de tal forma que ese fin en concreto es más fuertemente deseado en el futuro. Esto, sin embargo, no refuta la idea de que el hombre, en todo caso, valora positivamente el tiempo. Al contrario, hace pensar que, como el valor que asigna el hombre al tiempo es siempre positivo, debe existir, por necesidad, otro componente en la valoración intertemporal que sea negativo para que el diferencial de valor entre un el bien presente y el bien futuro sea negativo (es decir, que se valore más el bien en el futuro que en el presente).

Por lo tanto, vemos que en toda valoración intertemporal hay al menos dos componentes valorativos: uno que se refiere a la valoración del tiempo por el hombre, y otro referido al momento concreto en que el sujeto prefiere usar ese bien. Esta es la distinción que hace Heberner (2011, pp. 46-55) en un brillante artículo, basándose en las enseñanzas de Fetter (1904, pp. 141-142). En su escrito explica cómo, en una valoración intertemporal, existen dos componentes claramente separables: el momento oportuno del uso de un bien (timing) y el descuento temporal ${ }^{12}$. Desde esta óptica, por tanto, parece correcto

${ }_{12}$ Como expone Heberner (2011, pp. 55 y ss.), esta distinción aclara la TPTP de tal forma que las críticas recientes de Murphy (2003) caen por su propio peso. No obstante, no aborda Heberner la crítica de Hülsmann (2002), que, después de reconocer las bondades de la exposición contrafáctica misiana de la TPTP respecto a las exposiciones anteriores, rechaza que la preferencia temporal sea la verdadera causa del interés, dado que sólo puede explicar una acción (el hecho de desprenderse de la disponibilidad 
y necesario distinguir entre la preferencia temporal y la más concreta preferencia temporal pura (la que se refiere, únicamente, al valor que asigna el hombre al tiempo). Descuidar esta simple distinción fetteriana ha llevado a estériles debates ${ }^{13}$ sobre la posibilidad, entre otras cuestiones, de que los sujetos valoren más intensamente los bienes futuros que los presentes.

\section{c) Intercambios intertemporales en dinero e interés monetario}

Expuesta esta distinción, cabría preguntarse si la idea de que un bien presente es más valorado - en igualdad de circunstanciasque ese bien en el futuro tiene alguna aplicación o es totalmente inútil. Si en todas las valoraciones intertemporales entre bienes puede existir el componente de timing, no tendría sentido seguir formulando la TPTP utilizando la expresión caeteris paribus: de manera más precisa, habría que especificar que la teoría se refiere, únicamente, al componente del descuento temporal (anulando el otro componente). Si esto es cierto, bastaría enunciar la TPTP diciendo que el hombre valora el tiempo y que ello se manifiesta en todas las valoraciones intertemporales. No obstante, si bien es cierto que en casi todos los intercambios intertemporales aparecen los dos componentes valorativos que Fetter distinguía, existe al menos un bien en el que el componente de timing está muy atenuado (es decir, anulando el componente del descuento temporal, al sujeto le

de dinero para pagar los precios de los factores de producción), cuando el problema fundamental del interés consiste en la relación sistemática de esa acción con una segunda acción (concretamente, la venta del producto). Ello le lleva a concluir que el interés no es sino la diferencia que siempre se da entre el valor que el actor atribuye a sus fines y el valor de los medios que emplea para alcanzar tales fines. Aunque consideramos que tendría gran importancia responder a esta crítica, baste aquí con señalar que consideramos que Hülsmann —al igual que la tradición fisheriana que señala la productividad marginal como una de las causas del interés - confunde la causa de la existencia del interés originario con su surgimiento efectivo, dos cuestiones de naturaleza radicalmente distinta. A pesar de que autores como Kirzner (1993) o Garrison (1988) han separado ambas cuestiones con entera claridad, convendría a los defensores de la TPTP a responder específicamente a la crítica de Hülsmann en este sentido.

${ }^{13}$ En el siguiente epígrafe, como hemos adelantado, trataremos en detalle uno de estos debates (aunque, en este caso, sería injusto juzgarlo estéril); concretamente, el que tuvo lugar entre Hayek y Sraffa en los años 30. 
es prácticamente indiferente usar dicho bien en las condiciones presentes que en las futuras, merced a la estabilidad en el uso que ofrece este bien), y por lo tanto la locución caeteris paribus no es ya tan problemática. Este bien es el dinero. Como declara Heberner citando a Fetter:

Fetter recognized two cases of intertemporal differences in value: a present good trading for a future good and present money trading for future money. Time discount, which is the basis for interest operates in both cases, but another factor operates in the case of goods, namely, the timing of the use of the good. The sum of the two operating factors is time value.

(HEBerNer 2011, p. 46)

Aunque no es del todo cierto que en el dinero no aparece el componente de timing, ya que este se concreta en la expectativa de cambio en su poder adquisitivo, sí podemos afirmar que una unidad monetaria presente es más valorada que la misma unidad en el futuro eliminando el atenuado componente de timing (o si se prefiere, en igualdad de circunstancias). Vemos, por tanto, como la TPTP, que se basa en el indiscutible hecho de que el hombre valora el tiempo positivamente, puede explicar el diferencial valorativo positivo que siempre existe entre dos unidades monetarias si se asume que éstas tienen un poder adquisitivo constante (o dicho de otro modo, si anulamos el componente de timing). No es el objeto de este trabajo explicar in extenso cómo las especiales características del dinero llevan al hombre a considerar que el componente de timing en un intercambio intertemporal dinerario es fácilmente aislable. Baste aquí señalar cómo el dinero sirve al hombre de reflejo mediato del conjunto de fines presentes que desea satisfacer (además de la propia satisfacción del fin concreto que consiste en hacer frente a posibles fines que surjan en un futuro cercano). Como venimos argumentando, si el hombre tiene la posibilidad de satisfacer ahora un fin deseado inmediatamente, deberá ofrecérsele algo más del valor que asigna a dicho fin satisfecho en el presente para que renuncie a satisfacerlo en este momento. Esa diferencia de valor entre la satisfacción inmediata y aquello que se le ofrece al sujeto para que renuncie a la inmediatez de la satisfacción es lo que denominamos tipo de interés puro, y es, por necesidad lógica, siempre positivo 
cuando que se da un intercambio de este tipo. Si aplicamos dicha idea al dinero, vemos que el valor que el hombre da al hecho de tener dinero bajo su control procede de la capacidad que éste tiene para satisfacer un amplio número de necesidades presentes. Por lo tanto, el dinero (o, mejor dicho, la disponibilidad de dinero) tiene la capacidad de satisfacer un conjunto más o menos determinado de necesidades presentes. Es interesante recalcar que no es el uso del dinero presente frente al uso del dinero futuro lo que el actor tiene en mente en un intercambio intertemporal dinerario, sino la pérdida de disponibilidad de dinero presente frente a la disponibilidad futura ${ }^{14}$. Como decimos, el dinero disponible permite satisfacer los deseos más cercanos al momento presente, y al renunciar a esa disponibilidad se está renunciando a la posibilidad de que el sujeto haga frente a un mayor número de necesidades cercanas al momento presente, por lo que el actor preferirá el dinero disponible en el presente a la misma cantidad de dinero disponible en el futuro $^{15}$. Esta aclaración es relevante ya que las exposiciones confusas de la TPTP han llevado a críticos keynesianos, e incluso a autores identificados con la escuela austriaca como Murphy (2003, pp. 127-177), a afirmar que el interés es un fenómeno puramente monetario y que nada tiene que ver con la posposición del consumo, sino con la pérdida de disponibilidad del dinero. Aunque admitiendo que esto tiene un fondo de verdad, vemos cómo es una afirmación parcial y no del todo correcta, y cómo es posible retrotraerse a las causas, más fundamentales, por las cuales la pérdida de disponibilidad del dinero origina los tipos de interés monetarios. Entendemos ahora, por tanto, que, a pesar de que la preferencia temporal pura se manifiesta más claramente en el dinero que en otros bie-

${ }^{14}$ Rothbard era plenamente consciente de este hecho. Como aclara en una nota a pie de página en su exposición relativa a la preferencia temporal: «It is not valid to object that some might prefer to use the money in the future rather than in the present. That is not the issue here, which is one of availability for use. If a man wants to "save" money for some future use, he may "hoard" it rather than spend it on a future good, and thus have it always available». (Rothbard 2004, p. 386).

${ }^{15}$ Como nota aclaratoria, quizá conviene apuntar que a los tipos de interés que nos referimos son los tipos de interés monetarios puros. No tenemos en cuenta, por tanto, la prima de riesgo y la que recoge las expectativas de cambio del poder adquisitivo de la moneda. 
nes, el interés no es un fenómeno exclusivamente monetario. En lo sucesivo, veremos cómo es esta idea la que permite en la TACE yuxtaponer los cambios en los tipos de interés con los cambios en la estructura productiva.

III

\section{UNA REVISIÓN DEL DEBATE ENTRE HAYEK Y SRAFFA}

Una vez separados los diferentes componentes de las valoraciones intertemporales, estamos en posición de abordar el célebre debate entre Friedrich Hayek y Piero Sraffa sobre ciertos extremos de la exposición de la TACE que Hayek desarrolló en Prices and Production (1967 [1931]) ${ }^{16}$. De todos esos extremos, a los efectos de este escrito nos interesa únicamente la cuestión de la posible existencia de diversos tipos de interés naturales en los diferentes mercados intertemporales de bienes ${ }^{17}$. Este debate ha sido recientemente

${ }^{16}$ Sraffa (1932a), respondiendo a Hayek (1967 [1931]), negó la existencia de un tipo de interés natural único en una economía no monetaria y, por tanto, puso en tela de juicio la referencia de un (único) tipo de interés que Hayek utilizó en sus conferencias para desarrollar la TACE. Hayek (1932) contestó a las objeciones de Sraffa y, por último, éste (Sraffa 1932b) puso en entredicho la fortuna de esa contestación y, más generalmente, la validez de la TACE. Se pueden consultar resúmenes comentados de este debate en Lachmann (1986) y en Murphy (2010), textos a los que nos referiremos en el cuerpo de este escrito. Es igualmente interesante la discusión de Glassner y Zimmer$\operatorname{man}(2014)$.

${ }^{17}$ Nótese que en este epígrafe pretendemos demostrar la inexistencia de diversos tipos de interés puros según el tipo de bien presente que se intercambia intertemporalmente (es decir, que el tipo de interés será el mismo en un intercambio temporal, por ejemplo, entre unidades de dinero presentes y futuras, y en otro intercambio temporal, por ejemplo, entre kilogramos de trigo presentes y futuros) o por qué la noción de ownrates of interest, usando el nombre con el que los acuñó Keynes en su General Theory (1936), no son tipos de interés puros según la TPTP y por ello no deben ser incorporados por la TACE, contra lo que argumenta Murphy (2010). Pero no entramos a discutir la diferente cuestión de la posibilidad de varios tipos de interés entre las diferentes clases bienes futuros por razón de su vencimiento (por ejemplo, una clase de bien futuro por razón de su vencimiento sería recibir dinero dentro de un año y, otra distinta, recibirlo dentro de diez años). La existencia de diversos tipos de interés entre estos bienes, aun habiendo sido agotadas las posibilidades de arbitraje intertemporal, sería posible si los diferentes bienes futuros no fuesen perfectamente sustituibles entre sí, como ocurre fuera del equilibrio misiano de la EGU; de esta cuestión nos ocuparemos en el epígrafe IV. En este epígrafe III, entonces, siempre que hablemos de la inexisten- 
comentado por Robert P. Murphy (2010), quien ha expresado su preocupación por la insuficiente respuesta de los economistas austriacos a las críticas de Sraffa y la necesidad de reformar la exposición tradicional de la TACE a la luz de dichas críticas. Asimismo, ha criticado el tratamiento de Ludwig M. Lachmann (1986) sobre este asunto, calificándolo de retroceso. Nuestro estudio de este importante debate - y más concretamente el reciente tratamiento del mismo por parte de Murphy - revela la necesidad de, por un lado, separar con mayor precisión los diferentes componentes de las valoraciones intertemporales tal y como hemos propuesto en el epígrafe anterior siguiendo a Heberner (2011), y por otro, aclarar cómo la TACE no sólo es aplicable cuando existe un único tipo de interés de referencia en una EGU, sino igualmente cuando existen múltiples tipos de interés y la economía está en desequilibrio. Sólo clarificando estas dos cuestiones podremos blindar la TPTP y la TACE frente a críticas comunes a las mismas y disipar las confusiones que su tratamiento tradicional pueda ocasionar.

\section{El origen del debate}

El debate entre Hayek y Sraffa se centró en la tal vez problemática referencia teórica que Hayek (1967) utilizó en su desarrollo de la TACE. Hayek, en sus conferencias, trató de explicar la posibilidad de que los bancos privados induzcan al error generalizado a los agentes económicos en una economía monetaria tras la expansión crediticia y la subsecuente bajada del tipo de interés. En una economía monetaria, exponía Hayek, el interés monetario (o real) puede divergir del tipo de interés natural (o de equilibrio) porque la demanda y la oferta de ahorros no aparecen en su forma natural sino en forma de dinero. Como en una economía monetaria, los bancos tienen la capacidad de modificar la cantidad de dinero arbitrariamente, los agentes de la economía pueden creer erróneamente que

cia de diversos tipos de interés puros para cada bien, estaremos comparando distintos bienes presentes pero idénticos bienes futuros por razón de su vencimiento - por ejemplo, que ambos, dinero y trigo, se reciban a un año, o que ambos se reciban a dos años; pero no que el dinero se reciba a un año y el trigo se reciba a dos años). 
el cambio se debe a un aumento real de recursos disponibles, lo que puede desembocar en $\mathrm{crisis}^{18}$. En su réplica, Sraffa arguyó que en una economía no monetaria no hay un único tipo de interés natural, sino que existen tantos tipos de interés naturales como bienes presentes y futuros se intercambien. Sólo en el caso de que esa economía estuviese en equilibrio, decía Sraffa, existiría un tipo de interés único ${ }^{19}$. Cabe reparar, antes de nada, que en un primer momento Sraffa no juzga la idea que es en realidad el núcleo de la TACE, esto es, la posibilidad de que los bancos induzcan generalizadamente a error o no (como sí hará más adelante y, como veremos, errando en tal intento); aquí sólo pone en cuestión la referencia teórica que presenta Hayek para explicar el primer efecto derivado de la acción arbitraria de los bancos, y es en esto en lo que acaba centrándose el debate. Si esta referencia no fuese única, cree Sraffa, no tendría sentido el resto del discurso de Hayek. En este punto, nuestro examen del debate se bifurca en dos partes distintas: de un lado, tratar de verificar si es cierto que la referencia de Hayek es teóricamente errónea (esta cuestión fue ya resuelta por Lachmann, como a continuación veremos); y de otro lado, confirmar si la inadecuación a la realidad de la referencia a un tipo de interés único pone en peligro la TACE (cuestión a la que nos ocupamos en el apartado dedicado a la crítica de Murphy y completamos en el epígrafe IV).

\section{El acierto de Lachmann o el equilibrio inter-mercado}

El análisis de Lachmann (1986) aportó claridad y contribuyó a la cuestión de la posibilidad de que haya varios tipos de interés dependiendo del tipo de bienes que se intercambie intertemporalmente. La principal observación de este autor es que lo verdaderamente

\footnotetext{
18 «In a money economy, the actual or money rate of interest ("Geldzins") may differ from the equilibrium or natural rate, because the demand for and the supply of capital do not meet in their natural form but in the form of money, the quantity of which available for capital purposes may be arbitrarily changed by the banks». (Hayek 1967, p. 23).

19 «[T] but there might be at any one moment as many "natural" rates of interest as there are commodities, though they would not be "equilibrium" rates». (Sraffa 1932a, p. 49).
} 
problemático del debate es la diferente concepción de equilibrio que utilizaban Hayek y Sraffa y la incapacidad de ambos para identificar una tercera noción de equilibrio inter-mercado que resolvía la mayor parte de las confusiones del debate ${ }^{20}$; mientras que Hayek (1931), siguiendo a Wicksell, se refería al tipo de interés (de equilibrio) que resultaba del juego que se daba entre la demanda y la oferta de ahorros reales ${ }^{21}$, Sraffa (1932) respondió a Hayek desde la óptica del equilibrio neorricardiano, aduciendo que sólo podría darse un único tipo de interés (de equilibrio) si los precios spot de todos los bienes se igualasen con los precios forward ${ }^{22}$.

Así, se puede comprobar que los diferentes significados que los autores dan al concepto de tipo de interés de equilibrio es la manifestación más clara de las distintas nociones de equilibrio empleadas por cada autor, como señala Lachmann. Sin embargo, no es éste el único problema terminológico del debate. Para ambos autores, el tipo de interés natural es la diferencia valorativa entre el precio presente de un bien y el precio esperado del mismo en un futuro. Pero como hemos expuesto en detalle en el epígrafe II, a la luz de la TPTP sabemos que no todo margen valorativo que aparece entre un bien presente y el mismo en el futuro es un tipo de interés puro ${ }^{23}$.

${ }^{20}$ "[W]hen Hayek and Sraffa use the word "equilibrium" they use it to denote quite different things. For Hayek it means market-clearing demand-and-supply equilibrium, for Sraffa long-run cost-of-production equilibrium. Neither is ready to consider other kinds of equilibrium, for example, an inter-market equilibrium involving equality of interest rates in various commodity markets». (Lachmann 1986, pp. 153-4).

${ }^{21}$ «If it were not for monetary disturbances, the rate of interest would be determined so as to equalise the demand for and the supply of savings». (Hayek 1931, p. 23).

22 «In equilibrium the spot and forward price coincide, for cotton as for any other commodity; and all the "natural" or commodity rates are equal to one another, and to the money rate. But if, for any reason, the supply and the demand for a commodity are not in equilibrium (i.e. its market price exceeds or falls short of its cost of production), its spot and forward prices diverge, and the "natural" rate of interest on that commodity diverges from the "natural" rates on other commodities». (Sraffa 1932, p. 50). Este tipo de equilibrio neorricardiano, como comenta Murphy (2010, p. 8), es en realidad similar al de la EGU.

${ }^{23}$ Esta última frase es una paráfrasis de una conclusión de Roger Garrison (1976, p. 146) cuando dice, en una discusión diferente, que «all that need be recognized is that all rates are not rates of interest» (cursiva en el artículo original). Aquí Garrison elocuentemente traza la diferencia entre las tasas de crecimiento y el tipo de interés para responder a los intentos de contraejemplos como el de los higos de Fisher, que insatisfactoriamente tratan de demostrar que los tipos de interés negativos son posi- 
Si tenemos en cuenta la distinción fetteriana entre preferencia temporal y preferencia temporal pura para explicar los diferentes componentes en las valoraciones intertemporales, esta primera cuestión del debate queda resuelto. Dice Sraffa que para que existiese ese único tipo de interés entre todos los bienes, los precios spot de todos ellos deberían igualarse a sus precios forward; si el tipo de interés natural expresa la preferencia temporal y no la preferencia temporal pura, Sraffa no yerra al hacer tal afirmación. Pero es especialmente reprochable a Hayek, y no a Sraffa, el no haber aclarado en su respuesta que el tipo de interés desde el punto de vista de la TPTP no comprende el diferencial entre precios spot y precios forward. De hecho, llega afirmar explícitamente que el tipo de interés natural para cada bien es aquel al que se llega como resultado, no sólo de los factores que normalmente determinan el tipo de interés (puro), sino también de los factores que afectan a la demanda presente y futura de esos bienes ${ }^{24}$. Por lo tanto, el problema terminológico no sólo se reduce a la diferente concepción de equilibrio que ambos autores tienen, como hizo notar Lachmann, sino también a la incapacidad de Hayek de explicar que el tipo de interés al que se refiere la TACE nada tiene que ver con el diferencial entre el precio presente de un determinado bien y su precio esperado en el futuro como consecuencia de las expectativas en su demanda y oferta futura. Sin esta aclaración, difícilmente pueden entender los economistas no austriacos qué quería decir Hayek con la divergencia entre tipo de interés natural y el tipo de interés monetario, y cómo ésta podría desembocar en la ilusión de creer que se dan unas condiciones de abundancia en

bles. Nosotros, sin embargo, nos referimos al hecho más particular de que no en toda valoración intertemporal aparece la aislada la preferencia temporal pura que los partidarios de la TPTP señalamos como causa necesaria y suficiente del fenómeno del interés. Separar con precisión los componentes de las valoraciones intertemporales restulta necesario para no caer en las confusiones que este debate ha generado en torno a la TPTP y las implicaciones de esta teoría en la TACE.

${ }^{24}$ «I think it would be truer to say that, in this situation, there would be no single rate which, applied to all commodities, would satisfy the conditions of equilibrium rates, but there might, at any moment, be as many "natural" rates of interest as there are commodities, all of which would be equilibrium rates; and which would all be the combined result of the factors affecting the present and future supply of the individual commodities, and of the factors usually regarded as determining the rate of interest». (Hayek 1932, p. 245, nuestra cursiva). 
la economía que en realidad no existen. Por fortuna, Lachmann (1986) resolvió buena parte de la confusión cuando introdujo el concepto de equilibrio inter-mercado al que nos hemos referido. Este equilibrio no es otro que aquél donde, aun no alcanzándose el equilibrio neorricardiano de Sraffa (donde los precios spot y forward coinciden), se asume que se han agotado las oportunidades de arbitraje que surgen entre los mercados intertemporales de distintos bienes. Pero para que se alcance esta situación y exista un único tipo de interés (puro) uniforme en todos los mercados de bienes, Lachmann puntualiza, no es necesario que desaparezcan las diferencias entre los precios spot y forward de los distintos bienes; lo único necesario es aislar del tipo de interés único el componente de las expectativas del precio futuro (o siguiendo la terminología propuesta en el epígrafe anterior, separar la preferencia temporal pura del componente de timing). Así, como sostiene Lachmann, se resuelve el problema:

In a barter economy with free competition commodity arbitrage would tend to establish an overall equilibrium rate of interest. Otherwise, if the wheat rate were the highest and the barley rate the lowest of interest rates, it would become profitable to borrow in barley and lend in wheat. Inter-market arbitrage will tend to establish an overall equilibrium in the loan market such that, in terms of a third commodity serving as numéraire, say steel, it is no more profitable to lend in wheat than in barley. This does not mean that actual own-rates must all be equal, but that their disparities are exactly offset by disparities between forward prices.

(LACHMAN 1986, P. 154)

Esta forma elegante de solucionar el debate a través del concepto del equilibrio inter-mercado es otra forma de expresar lo que venimos defendiendo ${ }^{25}$. Nuestro análisis de la acción individualmente considerada donde diferenciábamos los componentes de las valoraciones intertemporales tiene su reflejo en este equilibrio general que propone Lachmann si separamos el diferencial de pre-

${ }^{25}$ Con la diferencia de que nuestro lenguaje pretende evitar las dificultades a las que nos referiremos más adelante que, desde el punto de vista praxeológico, se originan al hablar de tendencia al equilibrio. 
cios spot y forward del tipo de interés puro en aquella situación donde están agotadas las oportunidades de arbitraje intersectorial, por lo que una y otra perspectiva son en realidad la misma. Con esto queda claro, por tanto, que no toda diferencia entre las valoraciones intertemporales de un mismo bien constituye el tipo de interés al que se refiere la TACE cuando dice que éste refleja o no las preferencias temporales. De haber tenido en cuenta esta clarificación, Hayek fácilmente podría haberse centrado en el verdadero fondo del debate, esto es, el error generalizado al que pueden verse inducidos los agentes económicos si los bancos distorsionan los tipos de interés de tal forma que dejen de reflejar la preferencia temporal pura de los agentes del mercado.

\section{Una réplica a Murphy}

Murphy (2010) se lamenta de que Hayek no fue capaz de reconciliar las críticas de Sraffa con la TACE, de que los economistas austriacos han ignorado tales críticas, y además culpa a Lachmann de haber enmarañado el debate y de haber sido incapaz de resolver adecuadamente la objeción de Sraffa ${ }^{26}$. Creemos que es injustificado defender que Lachmann no dio luz a la discusión entre Hayek y Sraffa y que los austriacos deberían haber incorporado las objeciones de este último a la TACE. Es cierto que nadie puede negar la existencia de diferentes márgenes de precios presentes y esperados entre los distintos bienes (lo que Murphy llama own-rates of interest siguiendo la denominación keynesiana). Pero lo que sí podemos afirmar en virtud de la TPTP es que tales diferencias nada tienen que ver con la preferencia temporal pura que es la causa del fenómeno del interés, y que constituye la base de la TACE para explicar la acumulación de errores provocada por las acciones de bancos y autoridades monetarias que crean una falsa ilusión de abundancia de recursos. Por lo tanto, el escaso interés de los austriacos en

\footnotetext{
${ }^{26}$ «Hayek certainly did not fully reconcile his analysis of the trade cycle with the possibility of multiple own-rates of interest... Unfortunately... [a]s I will show in the case of Ludwig Lachmann... modern Austrians not only have failed to resolve the problem raised by Sraffa, but in fact no longer even recognize it». (Murphy 2010, p. 11).
} 
incorporar a la TACE la posibilidad de múltiples own-rates está más que fundada, puesto que reconocer su existencia en absoluto afecta a la TACE.

Pero cuando Murphy especifica cuál cree que es el verdadero error de Lachmann, la endeblez de su crítica se hace evidente. Según él, Lachmann tiene razón en que la tasa de rentabilidad en el equilibrio inter-mercado es la misma en todas las alternativas de intercambio intertemporal, al no existir oportunidades de arbitraje intersectorial. Pero afirma que, a pesar de ser esto cierto, Lachmann olvida que ese tipo de interés variará dependiendo de la unidad de cuenta (numéraire) que elija el observador (Murphy 2010, pp. 12-3 $)^{27}$. Tras ejemplificar su idea demostrando que, aunque en una misma situación de equilibrio inter-mercado el tipo de interés sí es el mismo una vez se ha elegido una unidad de cuenta (siendo ésta, por ejemplo, acero), éste variará si se cambia la unidad de cuenta (cambiando el acero, por ejemplo, por oro), concluye diciendo lo siguiente:

Lachmann's demonstration... does not establish what Lachmann thinks it does. The rate of return (in intertemporal equilibrium) on all commodities must indeed be equal once we define a numéraire, but there is no reason to suppose that those rates will be equal regardless of the numéraire. As such, there is still no way to examine a barter economy, even one in intertemporal equilibrium, and point to "the" real rate of interest. (Cursiva en el artículo original).

(MURPHY 2010, P. 14)

Podemos responder a estas críticas como lo habría hecho el propio Lachmann. El error fundamental de Murphy es creer en la ne-

${ }^{27}$ Esta observación de que el tipo de interés puede ser expresado en diferentes unidades de cuenta no es nada nueva. Como dicen Glassner y Zimmerman (2014, p. 20), se remonta a Fisher (1986) y aparece en Keynes (1936): «Fisher, himself, in Appreciation and Interest (1896) had introduced something like an own-rate analysis in discussing how the same real rate could be expressed equivalently as different nominal rates, depending on the choice of numeraire or unit of account. Keynes's analysis in chapter 17 of the General Theory is merely a generalization of Fisher's analysis, leading to a similar conclusion, that a unique real rate can be expressed equivalently in terms of many different nominal rates, each one depending on a different choice of numeraire or unit of account». 
cesidad de interpretar el interés como una herramienta estadística, como lo hace Sraffa, en vez de una abstracción teórica, que es como se usa en la TACE. Lachmann señala lo siguiente sobre esta cuestión en su libro Capital and its Structure (1978a):

[Sraffa] came to interpret Wicksell's «natural rate» as an average of «actual» own-rates as they would exist, side by side, in a barter economy, and not as the result of the operation of market forces. He thus substituted a statistical device for an analysis of market relationships. (Nuestra cursiva).

(LACHMANN 1978a, pp. 76-7)

Como hemos visto, Murphy admite el argumento de Lachmann de que en equilibrio inter-mercado hay un único tipo de interés y por lo tanto no tendría ningún sentido concebirlo como una media estadística $^{28}$. Sin embargo, vemos que lo que Murphy reprocha a Lachman es su incapacidad de ver que, incluso en equilibrio y con un único tipo de interés expresado en una determinada unidad de cuenta, pueden existir tantos tipos de interés como unidades de cuenta se utilicen — según la que el observador arbitrariamente elija. Vemos, por tanto, que al igual que Sraffa, ésta sigue siendo una crítica puramente estadística o empírica y no teórica, en tanto que la objeción se dirige a cómo identificar empíricamente el tipo interés natural de referencia; no a rebatir la lógica de la argumentación de la TACE (sobre cómo la acción de manipulación de los tipos de interés puede inducir a acumulaciones de errores empresariales). Por ello, pensamos que la crítica es del todo inválida ${ }^{29}$.

${ }^{28}$ Cabe notar aquí que, tal vez, está equivocada crítica fue propiciada por el confuso lenguaje de Hayek (1931), que en algunos fragmentos de su discurso parece hacer una recomendación de política económica en vez de centrarse en una descripción wertfrei de la TACE, señalando como referencia de tal política un tipo de interés que, en realidad, sólo se puede identificar mediante la abstracción teórica y no usando técnicas estadísticas.

29 Pero advierta el lector que, incluso pasando por alto la naturaleza empírica de la crítica a la TACE, tampoco es una crítica del todo correcta desde el punto de vista económico. Murphy asume en gran parte de su discusión que la unidad de cuenta puede ser escogida por el economista arbitrariamente. Pero lo cierto es que el mercado escoge su unidad de cuenta por las peculiares características del bien que acaba convirtiéndose en dinero en un periodo muy extendido de tiempo. Como dice el propio Lachmann en una discussion diferente: «Purely formal assumptions can vitiate 
A pesar de estas discrepancias, sí que compartimos con Murphy una importante conclusión a la que llega sobre el debate entre Hayek y Sraffa. En su respuesta, Hayek (1932, p. 246) propuso un ejemplo bastante aclaratorio que debería haber desarrollado con mayor profundidad. En éste, Hayek compara los diferentes efectos de un aumento en la oferta de dinero arbitrariamente realizado por los ban$\cos$ y los que tendría un aumento en la oferta del trigo («arbitrariamente» realizada por los granjeros). La conclusión a la que llega es que el aumento de la oferta de trigo haría que el tipo de interés de los préstamos en trigo disminuyese en comparación con el caso contrafáctico, es decir, en el cual los granjeros no hubiesen tomado tal decisión «arbitraria». Pero en ese caso, la bajada de interés de los préstamos en trigo no podría de por sí inducir a la acumulación de errores en la que se basa la TACE. Como explica Murphy con gran claridad:

In this tantalizing passage, Hayek puts his finger on the crucial point: When the commercial banks flood the loan market with artificial credits, this causes producers to erroneously begin projects that are physically unsustainable. Specifically, the producers lengthen production processes as if the savings of real goods had increased (when in fact they have not). Thus, when Hayek laments that the banks cause a divergence of the money from the equilibrium rate of interest, he is referring to the fact that the false interest rate disrupts

economic theories. The assumption, for instance, in monetary theory, that the market chooses "at random" one of its goods to serve as a numéraire contrasts with the plain fact that the precious metals were so chosen for very good reasons, and not at all "at random"». (Lachmann 1978b, p. 222). Y como hemos explicado en el epígrafe dedicado a la TPTP, las valoraciones intertemporales entre la unidad presente del bien dinero y la futura, en tanto que medio de intercambio, tienen muy atenuado el componente fetteriano de timing (que, a diferencia de lo que ocurre con otros bienes, puede aislarse fácilmente anulando las expectativas del cambio en su poder adquisitivo). Por otro lado, en aquellas economías donde existan varias monedas, el estadístico no tendrá problema en expresar el interés en las diferentes monedas si tiene en cuenta el poder adquisitivo relativo de cada una de ellas. En resumidas cuentas, en una economía monetaria, la unidad de cuenta del tipo de interés no habrá sido elegida arbitrariamente, por lo que la objeción que plantean Sraffa y Murphy no es, en realidad, tan problemática. Sólo en una economía no monetaria existiría el problema estadístico de elegir arbitrariamente una unidad de cuenta para expresar la abstracción teórica del interés; pero en tal caso dicho problema quedaría fuera del ámbito de la TACE, que explica la acumulación de errores de los agentes económicos como consecuencia de manipulaciones y distorsiones en la esfera monetaria (Rothbard, 1963, p. 6). 
the intertemporal coordination between producers and consumers. Sraffa clearly missed the entire essence of ABCT, because -as Hayek pointed out-Sraffa's suggested barter example would actually increase the subsistence fund; it was (by stipulation) a mistake, but only because consumers would have preferred that some other goods had been produced rather than the increment in wheat output. In other words, Sraffa's example of an erroneous (and unprofitable) increase in wheat production would not count as a «malinvestment» in the Misesian sense. (Cursiva en el artículo original)

(MuRphy 2010, p. 10)

Más tarde, para incorporar las críticas de Sraffa a la TACE, Murphy propone un equilibrio dinámico donde, a diferencia de lo que ocurre en la economía de giro uniforme (en adelante, EGU) y en el equilibrio neorricardiano, los precios spot van evolucionando con el paso del tiempo, aunque evolucionan de forma completamente predecible (Murphy 2010, p. 18). Según este autor, dicho equilibrio es más realista que la EGU e incluye la crítica de Sraffa sobre las diferencias intertemporales entre los precios presentes y esperados de los mismos bienes ocasionadas, no sólo por la preferencia temporal pura, sino también por las expectativas del cambio del valor de los bienes. No alcanzamos a atisbar ninguna razón que explique por qué Murphy opina que es necesario reformular la TACE asumiendo una perfecta capacidad de previsión de la evolución de los precios spot. Aunque efectivamente esto supondría un avance con respecto a la exposición centrada en la EGU, creemos que la literatura austriaca ya ha dejado muy atrás la necesidad del equilibrio dinámico que propone Murphy que incluye la perfecta capacidad de previsión. Por otra parte, el desvío argumentativo que toma Murphy después de señalar correctamente el fragmento donde Hayek vislumbra la verdadera solución al debate es sorprendente a la vez que desafortunado. Como dice Hayek en dicho extracto comentado por Murphy, lo que importa en realidad es el efecto que produce la intervención de los bancos y autoridades monetarias en la economía, y cómo existe la posibilidad de que junto a dicho efecto, aparezca un error generalizado que constituye el origen del ciclo $^{30}$. Si esto es cierto, es

${ }^{30}$ Un desarrollo de esta idea puede consultarse en Hülsmann (1998) y en el epígrafe IV de este escrito. 
irrelevante para la TACE la perfecta o imperfecta capacidad de previsión de los precios spot. Una vez reconocido el hecho de que las diferencias entre los precios esperados y los precios actuales de los bienes puede deberse no solamente a la preferencia temporal pura sino a la expectativa del cambio del valor del bien, no es necesario utilizar un presupuesto tan lamentablemente irreal como la perfecta capacidad de previsión para explicar las distorsiones bancarias que pueden generar la acumulación de errores que constituirán el origen del ciclo. Los actores del mercado podrán prever mejor o peor la evolución de los precios spot, pero una incorrecta previsión (asistemática) de los precios spot no puede generar ciclos. Lo crucial, como sostiene Hayek, es explicar qué puede inducir a los sujetos a errar de forma masiva, provocando la ilusión generalizada de que existen recursos reales cuando no los hay; o tomando el ejemplo de Hayek, por qué esto puede ocurrir con el bien dinero y no con el bien trigo.

En suma, queda claro cómo, a la luz de la TPTP, no existe una multiplicidad de tipos de interés (puros) entre los distintos bienes si están agotadas las oportunidades de arbitraje intersectorial. Por ello, tanto la crítica de Sraffa cuanto la propuesta de modificación de la exposición de TACE por parte de Murphy son infundadas. En el mejor de los casos, la crítica tiene una naturaleza estadística que, precisamente por tener esta naturaleza, no puede refutar la veracidad teórica de un tipo de interés único entre los distintos sectores de la economía.

IV

ESTRUCTURA DE TIPOS DE INTERÉS, DESEQUILIBRIO Y EL PATRÓN DE LA CURVA DE RENDIMIENTOS SEGÚN LA TACE

Hasta este punto hemos argumentado —en el epígrafe II- la conveniencia de enunciar la TPTP haciendo referencia explícita a los dos componentes de la valoración intertemporal (descuento temporal y timing) y ejemplificado la importancia de esta noción con el debate de Hayek contra Sraffa - en el epígrafe III. Asimismo, entendiendo que no toda diferencia entre el precio presente de un bien y su precio esperado constituye el tipo de interés puro al que 
se refiere la TACE, hemos rechazado la propuesta de Murphy de incluir en la exposición de ésta un equilibrio dinámico que haga frente a las objeciones de Sraffa. Pero hay al menos dos cuestiones que no sido tratadas hasta ahora y que nos proponemos estudiar en el presente epígrafe. Nos referimos, en primer lugar, a la cuestión de la posible existencia de diferentes tipos de interés en cada mercado de bienes futuros con diferente vencimiento (entendiendo que son distintos bienes futuros por razón de su vencimiento, por ejemplo, recibir una unidad monetaria en un año y recibir la misma en cinco años). Habiendo explicado en el epígrafe III la imposibilidad de que existan diferentes tipos de interés puros para distintos bienes si se agotan las oportunidades de arbitraje intermercado o intersectoriales, queda por tanto estudiar el supuesto en que se agotan las posibilidades de arbitraje inter-tiempo o intertemporales. La segunda cuestión que estimamos necesario desarrollar es la de si la referencia a un tipo de interés único (o a la tendencia al mismo) es esencial a la TACE, o dicho de otra forma, si esta teoría sigue siendo válida si la acción distorsionadora del sistema bancario y de las autoridades monetarias se diese en una situación, desde el origen, de desequilibrio (existiendo oportunidades de arbitraje intertemporales e intersectoriales ${ }^{31}$ ), y por tanto sin hacer referencia a un único tipo de interés (o a la tendencia al mismo). Por último, y partiendo de estas dos ideas previas, analizaremos las divergencias que determinadas acciones de los bancos privados y centrales pueden ocasionar en la estructura de tipos de interés; en la actual economía de intervencionismo monetario y dinero fiat, la estructura de tipos de interés nominales se suele alterar recurrentemente de una forma muy particular, y los movimientos observables de dicha estructura a lo largo del ciclo suelen seguir un mismo patrón, culminando éste normalmente en la inversión de la pendiente de la curva de rendimientos que anticipa la

${ }^{31}$ Advertirá el lector atento que esta cuestión engloba a la anterior. Como veremos, aun estando agotadas las oportunidades de arbitraje intertemporal en una economía diferente a la EGU, habrá varios tipos de interés y, por lo tanto, no será válida la referencia teórica de un tipo de interés único. La segunda cuestión de la que hablamos en el cuerpo del trabajo es aún más amplia que aquel primer escenario. Podríamos decir que la primera cuestiona la realidad o validez de la referencia a un tipo de interés único, mientras que la segunda trata sobre la esencialidad de dicha referencia. 
crisis. Este hecho ha sido observado y ampliamente tratado en la literatura mainstream, pero sólo ha encontrado una explicación satisfactoria de sus causas en la literatura austriaca.

\section{La curva de rendimientos y su pendiente}

Hemos visto cómo, según la TPTP, el hombre valora más una unidad monetaria presente que una unidad monetaria futura. Por tanto, no se debe descuidar qué tipo de dinero futuro (por razón del vencimiento) intercambian los agentes económicos por el dinero presente disponible, puesto que tal descuido puede conducirnos a conclusiones incompletas (sobre todo, en lo que respecta al desarrollo de la TACE). En efecto, es relevante el tiempo durante el cual los agentes económicos están dispuestos a perder la disponibilidad de su dinero presente. $\mathrm{Si}$, como hemos defendido, el tipo de interés expresado en dinero tiene por causa la preferencia temporal pura $\mathrm{y}$, más específicamente, la renuncia del propietario del dinero a la capacidad de hacer frente a las necesidades presentes que ese dinero posibilita, entonces el sujeto no valorará por igual la unidad monetaria futura y la unidad monetaria más futura (nos referimos, por ejemplo, a la unidad monetaria que se recibe a un año y la que se recibe a diez años). En virtud de la TPTP, por tanto, si admitimos que el hombre valora el tiempo, no sólo llegamos a la conclusión de que la unidad monetaria presente es más valorada que la futura, sino que también podemos afirmar que preferirá la unidad monetaria futura a la más futura ${ }^{32}$.

Hasta aquí sólo hemos posado nuestra atención en la preferencia temporal desde la perspectiva individual del hombre sin aludir a los instrumentos financieros particulares mediante los cuales se

${ }^{32}$ Bagus y Howden (2010, p. 72) exponen esta idea en terminos quizás más claros: «[C]eteris paribus, savers are only willing to forgo the availability of their funds for a longer period in exchange for a higher interest rate than is offered for short-termed investments. Imagine that there is option A to save and invest for 10 years risk-free with an annually compounded interest rate of 10 percent and option B to save and invest for 5 years risk-free with the option to renew the investment after 5 years for an additional 5 years with an equal annually compounded interest rate of 10 percent. Ceteris paribus, actors prefer option $\mathrm{B}$ ». 
realiza el intercambio intertemporal, lo cual es muy relevante; en el caso en que un actor esté dispuesto a renunciar a su dinero durante sólo un año, si asumimos la perfecta sustituibilidad de estos instrumentos financieros, el actor podrá, bien comprar un título de deuda a un año, bien comprar un título de deuda a cinco años (con el mismo tipo de interés) y, transcurrido un año, venderlo. De similar manera, si el actor quiere renunciar a su dinero durante dos años, tendrá las opciones de comprar un título a dos años, uno a cinco años (por el mismo interés) y venderlo en el mercado secundario al segundo año, e incluso la alternativa de comprar un título a un año y renovarlo por otro idéntico llegado el vencimiento. Pero este análisis sólo es válido cuando las condiciones del mercado y las preferencias intertemporales de sus agentes no varían con el tiempo. En la EGU, por ejemplo, las conclusiones a las que hemos llegado son válidas. En esta situación de equilibrio, necesariamente la curva de rendimientos debe ser completamente plana, ya que todos los instrumentos son perfectamente sustituibles ${ }^{33}$. Pero como apuntan Bagus y Howden (2010, p. 72), el EGU es un constructo tan irreal que es difícil aplicar las conclusiones que de él se derivan al mundo real. Y en efecto, en el mundo real las condiciones de la EGU no se dan. Rothbard falla en su análisis de la curva de rendimientos ${ }^{34}$ que se da en el mundo real precisamente porque da por hecho que en éste se dan condiciones que en realidad sólo existen en la EGU. Criticando nuestra posición, afirma que la idea de que pueda haber en el mercado varios tipos de interés para cada instrumento financiero de diferente plazo, «rests on the implicit assumption that individuals will be content to remain lenders in "shorts" at 5 percent while their fellow investors reap 7 percent» (Rothbard 2004, p. 447). Murphy responde desde la posición que aquí defendemos:

${ }^{33}$ Para ver una exposición enteramente basada en una situación de equilibrio de la EGU, o de la falsa creencia de que en el mundo real existe una tendencia hacia el mismo, ver Rothbard (2004, pp. 445-450). Para un análisis similar, ver Skousen (1990, pp. 193-194).

${ }^{34}$ La curva de rendimientos es aquella que relaciona los diferentes tipos de interés de instrumentos financieros con distinto plazo pero con idéntica calidad crediticia (por ejemplo, por provenir del mismo emisor). 
On the contrary, the [idea] rests on the implicit assumption that sometimes people will be in a situation where they can lend for a one-year term, but then they will not be net lenders the following period. For such people, the reason they are content in (say) year 1 to lend out at 5 percent, even though the five-year yield at that point is 7 percent, is that they need to spend that money in year 2. In order to reap the higher yield, they would need to part with their present goods for a longer term, and (for whatever reason) they are not prepared to do that. (Cursiva en el artículo original).

(MurPHy 2010, p. 26)

Por tanto, parece obligado admitir que, si nos alejamos de las condiciones de la EGU, la no sustituibilidad de los distintos bienes futuros con mismo riesgo hace que ofrezcan diferentes tipos de interés, incluso agotando las oportunidades de arbitraje entre ellos. En efecto, en el ejemplo de Murphy, el hecho de que los inversores en deuda a corto plazo reciban $5 \%$ de interés y otros inversores de deuda a largo plazo reciban $7 \%$ no quiere decir que los primeros estén desaprovechando una oportunidad de arbitraje. Simplemente no están dispuestos a renunciar a su dinero durante más tiempo para obtener una mayor rentabilidad, lo cual está en perfecta sintonía con la TPTP.

Por lo expuesto, hemos de admitir que en una economía sin giro uniforme, la pendiente de la curva de rendimientos es positiva si tenemos en cuenta la TPTP, sin incluir otros componentes valorativos incluidos en los tipos de interés nominales. Si además incluimos el componente de riesgo que incluyen los tipos de interés nominales, aunque la curva de rendimientos esté compuesta por títulos del mismo emisor, parece evidente que el riesgo crédito (o de impago) aumentará a medida que aumente el vencimiento de tales títulos ${ }^{35}$. En un entorno donde las expectativas de cambio del poder adquisitivo de la moneda sean constantes, por tanto, la pen-

${ }^{35}$ Este razonamiento es idéntico al seguido en Bagus y Howden (2010, pp. 70-1). Estos autores notan que con estas explicaciones a priori sobre la positividad de la pendiente de la curva de rendimientos se llegan a las mismas conclusiones de las teorías de la segmentación del mercado y de la preferencia por la liquidez, que estudian el fenómeno empírico de la positividad de dicha pendiente. Al no ser el objetivo de este trabajo estudiar con profundidad estas teorías, es conveniente a tal efecto referir al lector a Cwik (2003, pp. 53-79), donde sí se realiza este estudio. 
diente de la curva de rendimientos será positiva (si estas expectativas son inflacionarias, se hará más positiva; si son deflacionarias, la pendiente será menos positiva). Como veremos más adelante, la importancia de este hecho muestra la conveniencia de desagregar —aún más- el estudio de los precios relativos en la TACE; concretamente, los economistas austriacos tendrán que centrar su análisis cada vez con mayor detalle en el comportamiento de los diferentes tipos de interés (o precios resultantes de intercambios intertemporales) ${ }^{36}$.

\section{La problemática referencia al tipo de interés único}

Como hemos visto en el epígrafe III, dedicado al célebre debate entre Hayek y Sraffa, la referencia a un tipo interés único como base de la exposición en la TACE puede generar dudas sobre su validez. Aunque explícitamente Sraffa nunca hizo una crítica con verdadera naturaleza teórica, podemos reinterpretar su crítica y elevarla a una perspectiva más general y de mayor enjundia, de tal forma que cobre relevancia no empírica sino teórica. Esta reinterpretación se puede resumir en la siguiente pregunta: ¿en una situación

${ }^{36}$ Resaltando la importancia de la dimensión temporal del ahorro en la TACE se puede llegar a la conclusión de que las descoordinaciones intertemporales que estudia esta teoría pueden no sólo manifestarse debido a una insuficiencia de la cantidad de ahorro agregado disponible para cubrir los proyectos de inversión emprendidos como resultado de una expansión del crédito, sino también debido a un incorrecta adecuación entre el plazo del crédito y el tiempo que los ahorradores están dispuestos a renunciar al control efectivo de su dinero. Es decir, como expone Bagus (2010), no solo es importante la cantidad de ahorro agregado de la economía sino también la dimensión temporal de este ahorro. Este autor defiende que haber ignorado la importancia de la dimensión temporal del ahorro ha llevado, entre otras cosas, a que los economistas austriacos den por sentado que las crisis económicas se producen debido al funcionamiento de la reserva de banca fraccionaria, cuando este no es sino el caso más extremo de descalce de plazos. Sin entrar en la cuestión de si el descalce de plazos es legítimo o no - dos posiciones enfrentadas de este debate se pueden ver en Barnett y Block (2009a) y en Bagus y Howden (2009)—, sí compartimos con Bagus, al igual que con otros (Block y Barnett 2009b, Bagus y Howden 2010), la defensa de que la situación de descalce de plazos exagerado y generalizado puede igualmente generar ciclos económicos, y causar distorsiones en la estructura productiva, dando lugar a situaciones de iliquidez que impidan, en el advenimiento de la crisis, refinanciar operaciones con un mayor plazo que los préstamos obtenidos para financiarlas. 
en la que no exista un tipo de interés único (por ejemplo, aquella que hemos estudiado en el apartado inmediatamente anterior a éste), sigue siendo válida la TACE? O expresado de un modo más amplio, ¿nuestra teoría es incorrecta si se parte desde una situación de desequilibrio como generalmente se encuentra la economía? En la literatura austriaca se trata de buscar referencias al equilibrio - ya sea estático o dinámico- para explicar los efectos de la TACE. $Y$ en los casos en los que se admite que, efectivamente, en el mundo real la economía está en desequilibrio, se argumenta que existe una tendencia al equilibrio, permaneciendo inalterada la referencia teórica del tipo de interés único. Dado lo problemático del concepto de tendencia al equilibrio desde el método praxeológico ${ }^{37} \mathrm{y}$, además, el hecho de que en una economía sin giro uniforme existen varios tipos de interés (incluso agotadas las oportunidades de arbitraje entre los distintos bienes futuros), cabe analizar aquí si la TACE puede expresarse sin aludir a la tan problemática referencia del tipo de interés único.

La respuesta a esta cuestión es afirmativa. Como apuntamos arriba, Hayek (1932, p. 246) notó que el verdadero origen de los ciclos económicos era el error generalizado en el que caen los agentes del mercado, por lo que lo fundamental es estudiar cómo es posible que este error se verifique. La exposición tradicional de la TACE explica como el error es causado por una depresión del único tipo de interés, de tal manera que no refleja las preferencias temporales de la sociedad y, por lo tanto, ello induce a descoordinaciones intertemporales. Una cantidad ofertada de dinero presente superior a la que, en caso de no producirse la intervención de bancos y autoridades monetarias, habría existido, tiene como resultado, en igualdad de condiciones, un tipo de interés de equilibrio más bajo que el que habría resultado de no darse dicha intervención. Si ese cambio sucede de tal manera que los empresarios creen erróneamente que, la bajada del tipo de interés se debe a un aumento real de recursos disponibles (como consecuencia de la disminución del consumo), actuarán como si existiesen suficientes recursos para ter-

${ }^{37}$ Hülsmann ha expuesto, con impecable lógica, cómo desde un análisis praxeológico estricto no es posible hablar de una tendencia al equilibrio (Hülsmann 1997, pp. 29 y ss., 2000, pp. 16-7). 
minar sus proyectos. Si analizamos esta manera tradicional de describir el origen del ciclo, nos daremos cuenta que la referencia a un único tipo de interés no es necesaria. En dicha exposición, lo único que se dice es que se produce una determinada acción (de bancos o autoridades monetarias) que desencadena un determinado efecto (escenario real) que no se habría producido caso de no verificarse tal acción (escenario contrafáctico). Los agentes del mercado, interpretan erróneamente el cambio producido en el escenario real como si se hubiese producido una acción (aumento de los recursos reales disponibles) que, aunque tiene efectos similares a lo ocurrido en el escenario real, en realidad, no ha tenido lugar; por lo tanto, dichos agentes creen estar en un escenario diferente al real (escenario ilusorio). En suma, lo único necesario para exponer la TACE es advertir el carácter contrafáctico de las leyes económicas (relación entre el escenario real y el contrafáctico) y la necesidad de que se produzca una acumulación de errores para que tenga lugar el ciclo económico (relación entre el escenario real y el ilusorio).

\section{a) El carácter contrafáctico de la TACE}

Una vez reconocido el carácter contrafáctico de las leyes económi$\operatorname{cas}^{38}$ no es difícil entender que la referencia al tipo de interés único utilizado en las exposiciones tradicionales de la TACE, en realidad no es un elemento esencial a la misma. No es, por tanto, necesario, hacer referencia a un tipo de interés monetario único (o una tendencia al mismo) que, tras las intervenciones de los bancos privados y centrales, diverge del único tipo de interés natural. Perfectamente puede sustituirse por la idea más realista de que existe una determinada estructura de tipos de interés original que, como consecuencia de la acción de los bancos, pasaría a ser, en general, más baja que aquella que prevalecería en caso de no haberse producido tal acción (estructura de tipos de interés contrafáctica). Para explicar la naturaleza contrafáctica de la que hablamos, es ilustrativo citar un fragmento de la obra del profesor Huerta de Soto, introduciendo la idea de que existen varios tipos de interés en lugar de uno:

\footnotetext{
38 Para mayor detalle, ver Hülsmann (2003).
} 
[La] reducción de [los tipos de interés] del mercado crediticio... basta [que se verifique] al menos en términos relativos [o contrafácticos], es decir, en relación con [la estructura de tipos de interés] que habría preponderado en el mercado de no haberse verificado la expansión crediticia. Por eso es compatible incluso con que [la estructura de tipos de interés] en términos absolutos [o fácticos] suba, si es que sube menos de lo que se habría elevado en un entorno sin expansión crediticia (...); o con que, rebajándose [los tipos de interés], se reduzca[n] todavía más de lo que habría[n] bajado de no haberse producido la expansión crediticia. Por tanto, la reducción [de los tipos de interés] a la que nos estamos refiriendo es una realidad que nos explica la teoría y que habrá que interpretar históricamente teniendo en cuenta las circunstancias particulares de cada caso. (Cursiva en el fragmento original).

(Huerta DE SOTO 1998, p. 278).

Incluyendo la referencia de estructura de tipos de interés contrafáctica a la TACE, podemos resolver los dos problemas a los que nos estamos refiriendo en este apartado: en primer lugar, evitamos la problemática referencia al tipo de interés único; en segundo lugar, afirmamos que incluso aunque se parta teóricamente de situaciones de desequilibrio (y esta vez, sin hacer referencia al problemático concepto de tendencia al equilibrio), es posible exponer la TACE con éxito.

\section{b) Ciclos económicos como acumulaciones de errores}

Pero un cambio súbito en la estructura de tipos de interés que no corresponde con la contrafáctica no es lógicamente suficiente para producir el ciclo, entendido como acumulaciones de errores. Es necesario, además, que los actores crean que se dan unas condiciones que, en realidad, no se dan. Como declara Rothbard (1963) cuando investiga la verdadera naturaleza de las crisis económicas de carácter general (o que afectan a toda la economía), el economista debe preguntarse por qué se producen recurrentemente cúmulos de errores empresariales en toda la economía ${ }^{39}$. El principal problema a expli-

${ }^{39}$ En concreto, Rothbard (1963, p. 8) dice: «The main problem that a theory of depression must explain is: why is there a sudden general cluster of business errors? This is 
car por las teorías del ciclo es, por qué, de manera recurrente, llega un momento en que la gran mayoría de los empresarios se encuentra con pérdidas en sus balances. A este respecto, Hülsmann (1998), explica con mayor detalle cómo la TACE no necesita partir del irrealista presupuesto de que el hombre no yerra en condiciones normales (a diferencia de lo que hace Murphy al proponer su constructo de equilibrio dinámico como ya vimos), sino que basta con que explique por qué se origina un mayor número de errores que no se producirían si no se verificasen ciertos eventos ${ }^{40}$. Sin pasar por alto el esfuerzo empleado en clarificar que una teoría del ciclo no puede basarse en lo que él llama consecuencialismo ${ }^{41}$, nos interesa centrarnos en la particular teoría del ciclo de errores que propone. En particular, Hülsmann defiende que, para que se den las acumulaciones de errores de las que hablamos, deben existir instituciones (por ejemplo, la banca de reserva fraccionaria) que tengan la capacidad de generar errores independientemente del tiempo y del espacio. Estos errores generalizados, en realidad, no suponen otra cosa que la errónea creencia de los hombres en que dichas instituciones son aptas para alcanzar sus fines, cuando en realidad causan más perjuicio que beneficio. La conclusión a la que llega (si acaso más precisa, no tan distante a lo que en realidad defendían economistas como Mises y Rothbard) es que no son los propios cambios en las señales de mercado (concretamente, en la estructura

the first question for any cycle theory. Business activity moves along nicely with most business firms making handsome profits. Suddenly, without warning, conditions change and the bulk of business firms are experiencing losses; they are suddenly revealed to have made grievous errors in forecasting». (Cursiva en el fragmento original).

${ }^{40}$ En concreto, Hülsmann (2000, p. 2) señala la inflación como el evento que, según la tradiciónal TACE, produce el cúmulo de errores: «Austrian business cycle theory does not have to assume that, were it not for inflation, the market participants would not err at all. It can rely entirely on the idea that inflation causes additional errors; that is, more errors than otherwise would have occurred».

${ }^{41}$ Hülsmann (2000) explica con acierto la visión de que ninguna teoría puede ser válida si es consecuencialista, en el sentido de que considere que el error sea la consecuencia necesaria del cambio de las condiciones de la acción (en particular, la divergencia entre los tipos de interés nominales reales y los contrafácticos). Según este autor, ésta es una concepción falaz puesto que no tiene en cuenta la posibilidad de elección del hombre, que tiene la capacidad de correctamente interpretar los cambios en las condiciones de acción y, por tanto, anticipar sus efectos. 
de tipos de interés) las que determinan las acciones de los empresarios; es la creencia de éstos de que la razón subyacente que las altera es diferente a la que en realidad provoca tal alteración, y por ello caen masivamente en el error de creer que se dan unas condiciones de acción que, en realidad, no existen. Con esta exposición más precisa que la tradicional, resulta evidente que la referencia a un único tipo de interés es simplemente una herramienta teórica que, aunque es usada tradicionalmente por los economistas austriacos, no es esencial para exponer la TACE. Cualquier crítica a esta referencia teórica — como la de Sraffa- y no al verdadero núcleo de la TACE tal y como lo hemos expuesto, será por tanto una crítica accidental y no esencial a la teoría.

\section{Cambios en la estructura real de tipo de interés respecto a la contrafáctica}

Una vez aclarada la idea de que la propia depresión de la estructura de los tipos de interés no puede causar, por sí sola, ciclos económicos, sino que dicho cambio debe ser interpretado erróneamente por los agentes económicos, podemos abordar las diferentes formas en que la estructura de tipos de interés puede ser modificada por la acción de bancos o autoridades monetarias. En general, como venimos diciendo, las exposiciones tradicionales de la TACE han dependido de la referencia teórica de un único tipo de interés, sin estudiar detenidamente las diferentes formas que puede tomar la estructura de tipos de interés como consecuencia del funcionamiento de una institución defectuosa. Se asume, normalmente, que los tipos de interés a corto plazo tienen el mismo comportamiento que los tipos a largo plazo ${ }^{42}$. Por ello, el efecto que tiene una expansión monetaria según la mayor parte de las exposiciones tradicionales del ciclo es el de una bajada de la estructura de tipos de interés con respecto a la estructura contrafáctica, asumiendo que los tipos a corto plazo tienen el mismo comportamiento que los tipos

${ }^{42}$ A pesar de esto, hay exposiciones tempranas de la TACE, como la de Robbins (1971 [1934]), en la que se intenta explicar el diferente comportamiento de tipos a corto y a largo plazo a lo largo del ciclo. 
a largo plazo. Sólo recientemente la literatura austriaca ha empezado a fijar su atención en desagregar el diferente comportamiento de los tipos de interés nominales a corto y a largo plazo a lo largo del ciclo. En particular, otro posible movimiento de la curva que describe la literatura es el causado por el descalce de vencimientos. Como hemos explicado arriba, la curva de rendimientos es normalmente positiva. En este caso, al ser los tipos a largo plazo más altos que los tipos a corto, los intermediarios financieros pueden encontrar oportunidades de beneficio arbitrando la curva de rendimientos y haciéndola más plana. Si obtienen financiación barata (a corto plazo) y prestan a tipos de interés más altos, incrementan la demanda por los créditos a corto plazo y aumentan la oferta de los créditos a largo plazo. En consecuencia, los tipos de interés de los préstamos a largo plazo descenderán, mientras que aumentarán los tipos de interés de los préstamos a corto plazo, situación que no se habría verificado en caso de no haberse producido el descalce de plazos (es decir, la estructura de tipos de interés contrafáctica tendría una pendiente más positiva que en el caso en que se realiza el descalce de vencimientos). Si esto ocurre, la cantidad de ahorros a corto plazo dejará de fluir directamente a la estructura productiva, pasando indirectamente por un intermediario que transforma vencimientos; alargando el plazo de los préstamos pero, obviamente, sin alargar el plazo de los ahorros. Es decir, además de obtener una curva de rendimientos más plana, un segundo efecto es que, en la estructura productiva, el descalce de plazos provoca que los tipos de interés, de media, sean más bajos que en el supuesto contrafáctico en el que no hay descalce ${ }^{4344}$.

${ }^{43}$ Como apunta Davidson (2014, p. 83) de manera más concisa: «Because the amount of present money channeled into production by borrowers of short-term loans falls, while that channeled by borrowers of long-term loans rises, interest rates in the productive structure tend to be lower overall than they would be in the absence of [loan maturity mismatching]».

${ }^{44}$ Cabe señalar que en un entorno de dinero fiat y con bancos centrales que sistemáticamente intervienen en la esfera monetaria, la pendiente de la curva de rendimientos será, por lo general, mucho más pronunciada que en un entorno con patrón metálico y sin intervención. Debido a la extraordinaria caída del poder adquisitivo de la moneda en el primer entorno (en el que actualmente nos encontramos), las expectativas inflacionarias son mucho más acusadas que en el segundo, y por ello los tipos a largo plazo incorporan una prima de inflación que, a priori, no tendría por qué ser tan 


\section{El patrón de la estructura de tipos de interés durante el ciclo: la inversión de la curva de rendimientos y su explicación austriaca}

Acabamos de ver que no desemboca en ciclo económico toda divergencia entre la estructura de tipos de interés nominal y la que hubiese prevalecido en caso de que los bancos privados o centrales no la hubiesen modificado artificialmente; solo en el caso en que los agentes erróneamente crean que dicho cambio en los tipos de interés se produce por un aumento de los recursos reales disponibles y actúen en consecuencia emprendiendo proyectos excesivamente largos, puede darse la crisis. Pues bien: sentadas estas bases nos interesa analizar un aspecto concreto de la crisis, que es la evolución de los tipos de interés nominales a lo largo del ciclo. Hasta ahora, hemos estudiado dos posibles causas de los ciclos económicos, la inflación y el descalce de plazos, que tienen un distinto efecto en la estructura de tipos de interés. Pero interesa al economista del ciclo estudiar el comportamiento de la estructura de tipos de interés, no sólo en el origen del ciclo sino también, a lo largo del mismo. Este comportamiento es, ciertamente, observable, y hay abundante evidencia empírica sobre cómo la estructura de tipos de interés varía a lo largo del ciclo. Y en concreto, hay un fenómeno cuyas causas inquietan a economistas e inversores: la inversión de la pendiente de la curva de rendimientos que suele producirse antes de las recesiones económicas. A pesar de existir un amplio consenso entre economistas procedentes de las escuelas más diversas en cuanto a la existencia de una correlación entre la inversión de la curva de rendimientos y la inmediata recesión, existe bastante

abultada en un entorno no intervenido. Como comenta Murphy (2010, p. 29), este razonamiento a priori parece encontrar su respaldo empírico en Bordo y Haubrich (2004), donde se documenta que antes de los años 1910 los tipos de interés a corto plazo con frecuencia eran más altos que los tipos de interés a largo plazo, lo cual no es extraño si los agentes de los mercados de crédito tenían expectativas deflacionarias (lo cual no sería descabellado puesto que, como acreditan Friedman y Schwartz (1971, p. 15), durante el periodo entre 1865 y 1879 el nivel general de precios se redujo a la mitad en EE.UU.). Por lo tanto, al ser la curva de rendimientos más aplanada en un mercado no intervenido, hay menores oportunidades de arbitraje entre tipos a corto y a largo plazo, por lo que la probabilidad de hacer descender en exceso los tipos de interés en la estructura productiva, de tal forma que ello induzca al error, es mucho menor. 
confusión en cuanto a las causas que motivan este fenómeno, que es precisamente lo que nos interesa a efectos de este trabajo. Tras realizar un detallado resumen de la literatura no austriaca sobre la capacidad que tienen los movimientos en el diferencial de los tipos de interés por plazo de vencimiento ${ }^{45}$ para predecir recesiones y anticipar el crecimiento económico, Wheelock y Wohar (2009, p. 436) concluyen que, aunque la mayor parte de la literatura estudia empíricamente cómo dicho diferencial predice las recesiones, los economistas no han puesto tanta atención en explicar las causas de esta correlación. Otros exponentes de la materia (Estrella y Trubin, 2006, pp. 1-2), en cambio, defienden que sí hay suficientes teorías, la mayor parte de las cuales son compatibles entre sí, y que en conjunto sí son adecuadas para explicar el fenómeno que señalamos. Continúan Estrella y Trubin diciendo que el gran número de causas posibles - según se colige de su artículo, todas o al menos algunas de ellas reputadas suficientes para provocar la inversión de la curva, ya que unas pueden aparecer en ausencia de otrascomplica proponer una explicación única sobre este fenómeno. Pero esta multiplicidad de causas la entienden estos autores también como una explicación de por qué la relación entre la inversión de la curva y la posterior recesión es tan robusta estadísticamente. No obstante, si el economista austriaco efectúa una lectura sobre las causas que proponen estos autores ${ }^{46}$, enseguida reparará en lo insatisfactorias que en realidad son para explicar la inversión de la curva de rendimientos como hecho precedente de la recesión, ya que solo constituyen posibles causas inmediatas de la misma sin remontarse a las causas originarias ${ }^{47}$. En suma, la multiplicidad de

${ }^{45}$ Este diferencial o term spread constituye la diferencia existente entre los rendimientos de los bonos del Estado a corto y a largo plazo. Cuando esa diferencia se reduce hasta hacerse negativa se habla de inversión de la curva de rendimientos.

${ }^{46}$ No es el objetivo de este epígrafe argumentar pormenorizadamente la insuficiencia explicativa de las causas propuestas en la literatura para explicar el poder predictivo de la pendiente de la curva de rendimientos. El lector puede ver un resumen de las principales explicaciones de la correlación entre variación del term spread y las recesiones en Weelock y Wohar (2009, pp. 422 y ss.), Benati y Goodhart (2007, pp. 7 y ss.) y Estrella y Trubin (2006, p. 2).

${ }_{47} \mathrm{~V}$. gr., una de estas principales causas que la literatura propone para explicar el poder predictivo de dicho diferencial se refiere a las acciones de las autoridades monetarias: si éstas endurecen la política monetaria, haciéndola más contractiva, ello hará 
causas (aparentemente suficientes) propuestas por la literatura mainstream y su evidente inmediatez, hace pensar al economista conocedor de la TACE que, en realidad, existen causas subyacentes más fundamentales que explican de manera más satisfactoria el poder predictivo de la curva de rendimientos.

a) La conveniencia de una explicación con raíces austriacas. El enfoque de Cwik

Tradicionalmente los economistas austriacos no han prestado atención a la curva de rendimientos en sus exposiciones debido a la continua referencia al (único) tipo de interés de equilibrio presente en la EGU. Como hemos explicado anteriormente, en la EGU no cabe sino admitir que el arbitraje intertemporal hace que la curva de rendimientos sea aplanada (Rothbard 2004, pp. 445 y ss.; Skousen 1990, pp. 193-5). Pero tan pronto nos alejamos de este constructo imaginario, entendemos que la curva de rendimientos se hace positiva, al menos cuando las expectativas del poder adquisitivo de la moneda no son deflacionarias y en ausencia de distorsión generada por bancos privados o autoridades monetarias. Además, si se da esa distorsión de la estructura de tipos de interés por parte de bancos privados o centrales, la curva de rendimientos se revela como un importante indicador para determinar la fase concreta del ciclo en la que se encuentra la economía. Por estas dos razones, conviene incorporar a la TACE una exposición de los movimientos que generalmente realiza la curva de rendimientos a lo largo del ciclo. Pero también conviene a los autores no austriacos buscar las causas de estos movimientos en explicaciones que sólo la escuela austriaca puede proporcionar: en efecto, solo es posible encontrar

que los tipos de interés (a corto y a largo plazo) aumenten, subiendo relativamente más los tipos a corto plazo. Siendo esto cierto, es una explicación evidentemente parcial y que omite la situación en la que se encuentra la estructura productiva cuando los bancos centrales deciden endurecer la política monetaria ante el peligro de inflación de precios. Esto siempre ocurre después del periodo de política monetaria expansiva previa a la política contractiva de los bancos, por lo que quedaría pendiente la pregunta de si, en ausencia de política expansiva que se lleva a cabo en primer lugar, se produciría la inversión de la curva de rendimientos. 
una explicación válida de estos movimientos con una adecuada comprensión (a través del puente analítico de la TPTP) de cómo los cambios producidos en la estructura de tipos de interés pueden afectar a la estructura productiva, y de cómo ésta (tal y como enseña la TACE) va a sufrir un ajuste espontáneo cuando los agentes económicos empiecen a darse cuenta del error generalizado que constituye el origen del ciclo, lo cual, a su vez, va a tener un impacto de nuevo en la estructura de tipos de interés ${ }^{48}$.

Son varios los autores que ya han reparado en la conveniencia de incorporar la curva de rendimientos en la TACE (por ejemplo, Keeler 2001a, 2001b y Mulligan 2002, 2006) ${ }^{49}$. Sin embargo, la exposición más detallada de los efectos contrafácticos sobre la estructura de tipos de interés nominales y la estructura productiva que producen las inyecciones monetarias de crédito a corto plazo (en un primer momento, deprimiendo visiblemente la curva de rendimientos por su extremo de vencimientos a más corto plazo) la encontramos en Cwik (2004) ${ }^{50}$. Este autor analiza la evidencia, que

${ }^{48}$ Como Murphy nota: «mainstream economists are dimly aware of the connection between monetary (i.e. central bank) policy, short-term rates, and future economic growth. Yet because they lack the Austrians' sophisticated understanding of the capital structure, mainstream economists are reduced to saying that high interest rates stifle economic growth because of "reduced investment"». Murphy (2010, p. 33).

${ }^{49}$ Estos autores se atreven a decir que la diferencia observable entre los tipos de interés a largo plazo y a corto plazo es una buena representación empírica de la referencia teórica del tipo de interés único (wickselliano) y su efecto en la estructura productiva. Como expresan resumidamente Bismans y Mougeot (2009, p. 249), «Keeler (2001[a]) and Mulligan $(2002,2006)$ consider that "observed long-term rates are a good representation of natural interest rates, and especially that the slope of the yield curve represents the Wicksellian notion of the differential between market and natural rates" (p. 340)... [C]redit expansion lowers short-term rates higher than long-term rates. So, the term spread - long-term minus short-term interest rates- rises at the beginning of the artificial boom, then gradually decreases and becomes negative during the quarters just preceding a recession. In other words, the term spread inversions mark the turning points of the aggregate economic activity. When the term spread decreases, the structure of production becomes less roundabout as entrepreneurs reallocate resources away from production goods to consumption goods».

${ }^{50}$ Lamentablemente, este autor no tiene en cuenta en su argumentación el efecto que, a lo largo del ciclo, ocasiona el descalce de vencimientos sobre la estructura de tipos de interés como hemos descrito arriba (Cwik 2004, p. 120, nota 67), y tal y como han hecho otros autores austriacos - particularmente, Bagus (2010) y Bagus y Howden (2010). Generalmente se producen los dos efectos combinados, por lo que tener en cuenta ambos resulta necesario para explicar los movimientos de la curva de rendi- 
apunta a que en el decurso del ciclo los tipos de interés a largo plazo permanecen relativamente estables; son, en realidad, los tipos de interés a corto plazo los que varían a lo largo del ciclo ${ }^{51}$. Esto obliga a matizar la exposición tradicional de la TACE, dado que parece incontrovertible que, en un mercado de crédito altamente intervenido y que se sustenta sobre una base monetaria fácilmente manipulable de dinero fiat, el comportamiento de los tipos a corto no coincide con el de los tipos a largo desde el principio hasta el final del ciclo. En efecto, a principio del ciclo la evidencia apunta a que, tras la inyección de dinero en los mercados de crédito a corto plazo, disminuyen los tipos de interés a corto plazo sin disminuir los tipos a largo plazo. Esto se explica, según Cwik, por dos efectos simultáneos: en primer lugar, el efecto Wicksell (o desplazamiento uniforme de la curva de rendimientos hacia abajo, producido inicialmente por una primera depresión de los tipos a corto provocada por la inyección referida, y una posterior bajada de los tipos a largo y subida de los tipos a corto tras el arbitraje realizado por los bancos entre tipos a corto y tipos a largo) y, en segundo lugar el efecto Fisher (aumento de los tipos a largo debido a las expectativas de inflación de precios generadas tras las acciones de las autoridades monetarias ${ }^{52}$ ). Quizá esta explicación es criticable por ser especulativa - ise puede defender que en todo ciclo observado, el hecho de que los tipos a largo permanecen estables se debe a que el efecto Fisher contrarresta al efecto Wicksell en el extremo derecho de la curva?--, pero juzgamos indudable que tal y como está dise-

mientos a lo largo del ciclo. Esto no obstante, es interesante resumir las conclusiones a las que llega este autor con alguna aportación menor.

${ }^{51}$ Resume Murphy (2010, p. 33): «[T] he behavior of short-term rates fits with the Austrian verbal description. The short-term rate spikes before every recession, and then falls rapidly over the course of the recession and into the recovery phase. Another important observation is that the flattening yield curve before a recession typically occurs because the short rate jumps higher than the long rate, rather than the long rate falling below the short rate».

${ }^{52}$ Nótese que esto es contrario a lo que Mises decía sobre el lapso temporal que media entre la expansión monetaria y el aumento de prima por inflación: «The price premium always lags behind the changes in purchasing power because what generates it is not the change in the supply of money (in the broader sense), but the necessarily later occurring effects of these changes upon the price structure». Mises (1998, p. 542). 
ñada la política monetaria, las autoridades no tienen el control sobre los tipos a largo que tienen sobre los tipos a corto (lo cual en efecto, podría llevar a que los agentes no sólo incrementasen la prima por inflación en los créditos, sino también la prima de riesgo debido al mayor riesgo de impago en los créditos a largo plazo, algo del todo previsible en una economía que históricamente ha experimentado ciclos recurrentemente). Aun así, consideramos que otra explicación de este hecho empírico puede ser más razonable. Como hemos explicado con cierto detalle, una mera divergencia entre estructura de tipos observable y la que prevalecería en caso de no haberse producido la expansión crediticia, no puede por sí misma causar una crisis. Los empresarios deben creer erróneamente que esta bajada les permitirá embarcarse en nuevos proyectos sin encontrar ningún problema en el futuro cuando éstos se aproximen a su término (como si existiera un suficiente fondo de subsistencia de recursos reales que les permitiese acabar dichos proyectos). Ante el descenso de la estructura de los tipos de interés (en concreto, aquellos que son a corto plazo), el efecto capitalización hace que la productividad marginal estimada sea superior para los proyectos largos, lo cual no sólo atraerá los empresarios marginales (aquellos que no habrían aceptado los préstamos de no ser éstos más baratos), sino que nuevos empresarios en busca de beneficios aumentarán la demanda de crédito a largo plazo para financiar los nuevos proyectos de mayor longitud. En suma, otra posible explicación - si bien especulativa, quizás no tanto como la de Cwik- de la relativa estabilidad de los tipos de interés a largo plazo es el aumento de la demanda de la financiación a largo plazo como consecuencia del error masivo (aumento de la productividad marginal percibida en proyectos largos) que causa la crisis, a lo que podríamos denominar efecto ilusión de abundancia.

Pero dejando aparte las concretas formas en que se origina tal error, que incontrovertiblemente requeriría un examen más minucioso para llegar a conclusiones válidas, lo que es seguro es que a medida que la ilusión de abundancia se generaliza, más empresarios comienzan a invertir en proyectos excesivamente largos y, al propio tiempo, los receptores de las nuevas rentas (los propietarios de los factores originarios de producción) consumen más, relativamente y en términos reales, respecto a la situación contrafáctica. La 
mala inversión y el sobreconsumo, tal y como explica la TACE, llevan a un momento en que la situación comienza a revelarse insostenible. Es en este momento cuando la pendiente de la curva de rendimientos comienza a hacerse menos positiva hasta aplanarse o llegar a ser negativa. Hay según Cwik y a la luz de la TACE, dos posibles causas inmediatas de esto. Una es la contracción del crédito como consecuencia de un endurecimiento de la política monetaria ${ }^{53}$. Si, como decía Hayek (1967, p. 150), la única manera de que las autoridades monetarias consigan posponer el advenimiento de la recesión es expandir el crédito de forma constantemente creciente, al contraerse el crédito comenzará el reajuste espontáneo de la estructura productiva. La segunda causa no es otra que, a pesar de mantener la política monetaria relajada, se den espontáneamente los efectos de reajuste de la estructura productiva, que tenderá a adecuarse a las preferencias de los consumidores ${ }^{54}$. Cwik deja claro que, de acuerdo con la TACE, al contrario de lo que opinan los economistas no austriacos, de no haberse producido la primera, acabaría produciéndose irremediablemente la segunda. En ambos casos, ante la insuficiencia de fondos para acabar los proyectos empresariales y la alta demanda de créditos a corto plazo para refinanciar dichos proyectos, los tipos de interés a corto plazo suben hasta quedar la curva de rendimientos aplanada o incluso invertida (subida que puede agravarse si se reduce la oferta de préstamos a corto plazo). A este efecto descrito podríamos añadir una explicación análoga — si bien tal vez no simétrica- al efecto ilusión de abundancia antes descrito, que bien podríamos llamar efecto descreimiento (y que se concretaría en una disminución de la productividad marginal estimada para proyectos a largo plazo, disminuyendo la demanda de créditos a largo plazo para financiarlos que, en conjunción con la disminución de la oferta de estos créditos, en última instancia ocasionaría una relativa estabilidad en los tipos de interés a largo).

Este enfoque, como vemos, es ciertamente acorde con las enseñanzas de la TACE y es, por ello, el más satisfactorio hasta el mo-

${ }^{53}$ Como hemos mencionado ya al criticar las causas que proponen los autores no austriacos, esto es solamente una causa inmediata del cambio de pendiente de la curva.

${ }^{54}$ Ambas causas están explicadas con meridiana claridad en Huerta de Soto (1998, pp. 317-8). 
mento para explicar el patrón recurrente que la estructura de tipos de interés nominales suele seguir a lo largo del ciclo. Sin embargo, parece necesario incluir en el análisis un estudio más detallado del peculiar comportamiento de la estructura real de tipos interés con respecto a la estructura contrafáctica, así como del efecto concreto de la transformación de plazos efectuada por los bancos, que en un entorno intervenido será normalmente excesivo por existir instituciones que sistemáticamente lo fomentan (Bagus 2010, p. 13). Por todo lo explicado hasta ahora, este análisis más detallado del descalce de plazos y el refinamiento de la explicación de Cwik son una línea de investigación en la que los economistas austriacos deberían enfocar sus esfuerzos en el futuro para avanzar en el estudio de la TACE.

\section{CONCLUSIONES}

Son, fundamentalmente, dos los objetivos que nos hemos propuesto en este artículo. Primeramente, hemos buscado aclarar las confusas enunciaciones de la TPTP que han llevado a sus críticos a menospreciarla sin fundamento. En este sentido, hemos pretendido subrayar la importancia de la cabal comprensión de la TPTP para quien pretenda analizar críticamente la TACE, ya que solo entendiendo las causas reales del tipo de interés monetario es posible comprender cómo la manipulación de éste puede generar distorsiones en la estructura productiva. En segundo lugar, hemos explorado desde varios ángulos la idea de incorporar en la TACE múltiples tipos de interés sin hacer referencia a un tipo de interés único o a la tendencia al mismo. El desarrollo de estos objetivos lo podemos resumir con el siguiente recorrido amplio de vuelo por nuestro trabajo:

En primer lugar, hemos reafirmado la validez de la TPTP respondiendo a algunas de las críticas que esta teoría ha despertado. Para ello, hemos defendido, siguiendo a Heberner (2011), la necesidad de incluir en las formulaciones de la TPTP la distinción fetteriana de los dos componentes valorativos presentes en todas las valoraciones intertemporales (descuento temporal y timing). Seguidamente, y utilizando la sólida base de la TPTP, hemos 
analizado el debate entre Hayek y Sraffa sobre la posibilidad de múltiples tipos de interés cuando no han sido agotadas las oportunidades de arbitraje inter-mercado. Tras concluir con Lachmann (1986) que la referencia a un tipo de interés único es válida al menos en este aspecto, hemos criticado el planteamiento de Murphy (2010) que de manera innecesaria intenta incorporar a la TACE las inválidas críticas sraffianas que, siendo de naturaleza estadística, no pueden invalidar la referencia teórica de un tipo de interés único. A continuación, hemos abordado dos cuestiones: la primera versa sobre la realidad o validez de la referencia a un tipo de interés único (o sobre si existen múltiples tipos de interés cuando se han agotado las oportunidades de arbitraje intertemporal, que no inter-mercado) y, la segunda, sobre la esencialidad de la referencia a un tipo de interés único (o de si es posible formular la TACE sin hacer referencia a un único tipo de interés). En cuanto a la primera cuestión hemos concluido, apoyándonos en Bagus y Howden (2010) y en Murphy (2010), que, una vez el análisis se aleja de la EGU, la no sustituibilidad de los bienes futuros obliga a admitir que existen varios tipos de interés que, en virtud de la TPTP, serán tanto más elevados cuanto más lejano del presente sea el vencimiento. En cuanto a la segunda cuestión, hemos analizado cómo basta con reparar en la naturaleza contrafáctica de la TACE y en la condición necesaria de que haya errores generalizados y recurrentes para entender que la referencia teórica al tipo de interés único que utilizan las exposiciones tradicionales es, en realidad, accesoria. Por último, hemos explicado la conveniencia de desarrollar un análisis más desagregado de la TACE, haciendo referencia al diferente comportamiento de los tipos de interés a largo y a corto plazo en el decurso del ciclo. Para ello, hemos comentado con Cwik (2001) el patrón que la curva de rendimientos suele seguir a lo largo de los ciclos y las causas originarias del fenómeno de la inversión de la curva de rendimientos. 


\section{REFERENCIAS BIBLIOGRÁFICAS}

Bagus, P. (2010): «Austrian Business Cycle Theory: Are 100 Percent Reserves Sufficient to Prevent a Business Cycle?», Libertarian Papers, vol. 2, art. no. 2.

BAgus, P. y Howden D. (2009): «The Legitimacy of Loan Maturity Mismatching: A Risky, but Not Fraudulent, Undertaking», Journal of Business Ethics, vol. 90, no. 3, pp. 399-406.

- (2010): «The Term Structure of Savings, the Yield Curve, and Maturity Mismatching», Quarterly Journal of Austrian Economics. vol. 12, no. 3, pp. 64-85.

BarnetT, W. y Block, W. (2009a): «Time Deposits, Dimensions, and Fraud». Journal of Business Ethics, vol. 88, no. 4, pp. 711-716.

- (2009b): «Crash and Carry: Financial Intermediaries, the Intertemporal-Carry Trade, and Austrian Business Cycles», Etica y Politica / Ethics and Politics, vol. 11, no. 1, pp. 455-469.

BenAtI, L. y GoOdHART, C. (2007): «Investigating Time-Variation in the Marginal Predictive Power of the Yield Spread», European Central Bank Working Paper Series, no. 802, (August).

Bismans, F. y Mougeot, C. (2009): «Austrian Business Cycle Theory: Empirical Evidence», Review of Austrian economics, vol. 22, pp. 241247.

BLOCK, W. (1978): «The Negative Interest Rate: Toward a Taxonomic Critique», Journal of Libertarian Studies, vol. 2, no. 2, pp. 121-124. Bordo, M. y Haubrich J. (2004): «The Yield Curve, Recessions, and the Credibility of the Monetary Regime: Long Run Evidence 18751997», N.B.E.R. Working Paper, no. 10431 (April).

DAVIDSON, L. (2014): «The Economic Consequences of Loan Maturity Mismatching in the Unhampered Economy», The Quarterly Journal of Austrian Economics, vol. 17, no. 1, pp. 67-91.

Estrella, A. y Trubin, M. (2006): «The Yield Curve as a Leading Indicator: Some Practical Issues», Federal Reserve Bank of New York Current Issues in Economics and Finance, vol. 12, no. 5.

FetTER, F. (1904): Principles of Economics, Nueva York, The Century Co. FISHER, I. (1896): Appreciation and Interest, Nueva York, Macmillan.

FrIedman, M. y Schwartz, A.J. (1971): Monetary History of the United States 1867-1960, Princeton, Princeton University Press. 
GARRISON, R. (1979): «In Defense of the Misesian Theory of Interest», Journal of Libertarian Studies, vol. 3, no. 2 (141-150).

- (1988): «Professor Rothbard and the Theory of Interest», En Block, W. y RocKWelL, L.H. (1988): Man, Economy and Liberty: Essays in Honor of Murray N. Rothbard, Auburn (Alabama), The Ludwig von Mises Institute.

Glassner, D. y Zimmerman, P.R. (2014): «The Sraffa-Hayek Debate on the Natural Rate of Interest», Disponible en <http://ssrn.com/ abstract $=2221695>$.

Hayek, F. Von (1967 [1931]): Prices and Production, Nueva York, Augustus M. Kelly.

- (1932): «Money and Capital: A Reply», The Economic Journal, vol. 42, no. 166 (June): pp. 237-249.

Heberner, J.M. (2011): The Pure Time-Preference Theory of Interest, Auburn (Alabama), Ludwig von Mises Institute.

Huerta De Soto, J. (1998): Dinero, Crédito Bancario y Ciclos Económicos, Madrid, Unión editorial [ $5^{\circ}$ edición, 2011].

Hülsmann, J.G. (1997): «Knowledge, Judgment, and the Use of Property», Review of Austrian Economics, pp. 23-48.

- (1998): «Toward A General Theory of Error Cycles», Quarterly Journal of Austrian Economics, vol. 1, no. 4, pp. 1-23.

- (2000): «A Realist Approach to Equlibrium Analysis», Quarterly Journal of Austrian Economics, vol. 3, no. 4, pp. 3-51.

- (2002): «A Theory of Interest», Quarterly Journal of Austrian Economics, vol. 5, no. 4, pp. 77-110.

- (2003): «Facts and Counterfactuals in Economic Law», Journal of Libertarian Studies, vol. 17, no. 1, pp. 57-102.

KeELer, J. (2001a): «Empirical Evidence on the Austrian Business Cycle Theory», Review of Austrian Economics, vol. 14, no. 4, pp. 331-351.

- (2001b): «Relative Prices and the Business Cycle», Paper presented at the Southern Economic Association Annual Meetings in Tampa, the Society for the Development of Austrian Economics, Session 42C: Research in Empirical Austrian Economics.

KeYnes, J.M. (1936): The General Theory of Employment, Interest and Money, Londres, Macmillan.

KIRZNER, I.M. (1993): «The Pure Time-Preference Theory of Interest: An Attempt at Clarification», En HEBERNER, J.M. (1993): 
The Meaning of Ludwig von Mises: Contributions in Economics, Sociology, Epistemology, and Political Philosophy, Norwell (Massachusetts), Kluwer Academic Publishers.

LachmanN, L.M. (1978a [1956]): Capital and its Structure, Kansas City, Sheed Andews and McMeel.

- (1978b): «Vicissitudes of Subjectivism and the Dilemma of the Theory of Choice», En LAVOIE, D. (2005 [1994]): Expectations and the Meaning of Institutions, Nueva York, Routledge.

- (1986): «Austrian Economics under Fire: The Hayek-Sraffa Duel in Retrospect», En LAVOIE, D. (2005 [1994]): Expectations and the Meaning of Institutions, Nueva York, Routledge.

Mises, L. Von (1998): Human Action. A Treatise on Economics. The Scholar's Edition, Auburn (Alabama), Ludwig von Mises Institute.

Mulligan, R. (2002): «A Hayekian Analysis of the Term Structure of Production», Quarterly Journal of Austrian Economics, vol. 5, no. 2, pp. 17-33.

- (2006): «An Empirical Examination of Austrian Business Theory», The Quarterly Journal of Austrian Economics, vol. 9, no. 2, pp. 66-93.

MuRPHY, R.P. (2003): Unanticipated Intertemporal Exchange in Theories of Interest, Doctoral Dissertation, New York University, Disponible en <http://consultingbyrpm.com/uploads/Dissertation.pdf> (2010): «Multiple Interest Rates and the Austrian Business Cycle Theory», Disponible en <http://consultingbyrpm.com/uploads/Multiple\%20Interest\%20Rates\%20and\%20ABCT.pdf>.

Pellengahr, I. (1996): The Austrian Subjective Theory of Interest, Frankfurt, Peter Lang.

Reisman, G. (1996): Capitalism. A Treatise on Economics, Ottawa (Illinois), Jameson Books.

RobiIns, L. (1971 [1934]): The Great Depression, Nueva York, Freeport. Rothbard, M.n. (2004): Man, Economy and State, with Power and Market, Scholar's Edition, Auburn (Alabama), The Ludwig von Mises Institute [ $2^{\circ}$ edición, 2009].

- (1963): America's Great Depression, Auburn (Alabama), The Ludwig von Mises Institute [ $5^{\circ}$ edición, 2000].

SKousen, M. (1990): The Structure of Production, Nueva York, New York University Press. 
SRAFFA, P. (1932a): «Dr. Hayek on Money and Capital», The Economic Journal, vol. 42, no. 165 (March), pp. 42-53.

- (1932b): «[Money and Capital]: A Rejoinder», The Economic Journal, vol. 42, no. 166 (June), pp. 249-251. 